\title{
Enhanced glycolytic metabolism supports transmigration of brain-infiltrating macrophages in multiple sclerosis
}

\author{
Deepak Kumar Kaushik, ${ }^{1,2}$ Anindita Bhattacharya,, ${ }^{1,2}$ Reza Mirzaei, ${ }^{1,2}$ Khalil S. Rawji, ${ }^{1,2}$ Younghee Ahn, ${ }^{3,4}$ Jong M. Rho, ${ }^{3,4,5}$ \\ and V. Wee Yong ${ }^{1,2}$
}

${ }^{1}$ Hotchkiss Brain Institute, ${ }^{2}$ Department of Clinical Neurosciences, ${ }^{3}$ Alberta Children's Hospital Research Institute, ${ }^{4}$ Department of Pediatrics, and ${ }^{5}$ Department of Physiology and Pharmacology University of Calgary, Calgary, Alberta, Canada.

\begin{abstract}
The migration of leukocytes into the CNS drives the neuropathology of multiple sclerosis (MS). It is likely that this penetration utilizes energy resources that remain to be defined. Using the experimental autoimmune encephalomyelitis (EAE) model of MS, we determined that macrophages within the perivascular cuff of postcapillary venules are highly glycolytic, as manifested by strong expression of lactate dehydrogenase A (LDHA), which converts pyruvate to lactate. These macrophages expressed prominent levels of monocarboxylate transporter-4 (MCT-4), which is specialized in the secretion of lactate from glycolytic cells. The functional relevance of glycolysis was confirmed by siRNA-mediated knockdown of LDHA and MCT-4, which decreased lactate secretion and macrophage transmigration. MCT-4 was in turn regulated by EMMPRIN (also known as CD147), as determined through coexpression and co-IP studies and siRNAmediated EMMPRIN silencing. The functional relevance of MCT-4-EMMPRIN interaction was confirmed by lower macrophage transmigration in culture using the MCT-4 inhibitor $\alpha$-cyano-4-hydroxy-cinnamic acid (CHCA), a cinnamon derivative. CHCA also reduced leukocyte infiltration and the clinical severity of EAE. Relevance to MS was corroborated by the strong expression of MCT-4, EMMPRIN, and LDHA in perivascular macrophages in MS brains. These results detail the metabolism of macrophages for transmigration from perivascular cuffs into the CNS parenchyma and identify CHCA and diet as potential modulators of neuroinflammation in MS.
\end{abstract}

\section{Introduction}

Multiple sclerosis (MS) is a demyelinating and neurodegenerative condition associated with the migration of activated lymphocytes and macrophages into the CNS. These leukocytes enter the white matter by crossing the blood-brain barrier (BBB) in areas of inflammation called the perivascular cuffs (1-3), as well as through the CNS-meningeal barrier $(4,5)$. The accumulation and activation of leukocytes in areas of infiltration initiates a cascade of events leading to the loss of BBB integrity, which further aids the transmigration of leukocytes into the brain parenchyma $(3,6$, 7). Recent studies highlight the existence of a metabolic switch within leukocytes that intricately regulates their activation states by changing their metabolic profiles. For example, proinflammatory macrophages and $\mathrm{T}$ cells rely heavily on glycolysis, with a strong resemblance to the metabolic states of tumor cells $(8,9)$. In contrast, antiinflammatory macrophage subsets predominantly utilize oxidative phosphorylation for their functions $(9,10)$. In this regard, the metabolic requirement of leukocytes and its relevance to MS pathogenesis are not well understood.

Conflict of interest: The authors have declared that no conflict of interest exists. Copyright: @ 2019, American Society for Clinical Investigation.

Submitted: August 6, 2018; Accepted: May 16, 2019; Published: July 15, 2019.

Reference information: J Clin Invest. 2019;129(8):3277-3292.

https://doi.org/10.1172/JCl124012.
During glycolysis, a molecule of glucose generates 2 molecules of pyruvate, which enters the TCA cycle (oxidative phosphorylation) or is reduced under anaerobic conditions to lactate. Generation of lactate from pyruvate in normoxic conditions is referred to as aerobic glycolysis or the Warburg phenomenon, which is a well-known feature of tumor cells. The generation of lactate from pyruvate is driven by lactate dehydrogenase (LDH), which works at near-equilibrium thermodynamics (11). LDH functions as a tetramer of LDHA and LDHB subunits. While LDHB favors lactate-to-pyruvate conversion, LDHA is a rate-limiting enzyme with higher affinity for pyruvate, which it converts to lactate. This step is crucial for recycling of $\mathrm{NAD}^{+}$, a coenzyme important for the functioning of glyceraldehyde 3-phosphate dehydrogenase, another glycolytic enzyme. LDHA is strongly associated with aerobic glycolysis and is implicated in the initiation and maintenance of tumor cells $(8,12)$. It is crucial in the production of lactate, a monocarboxylic acid, that has the capacity to modulate innate and adaptive immune responses (13-15). Thus, interference with lactate machinery presents an attractive opportunity to treat immune-mediated disorders. Since LDH activity is not critical for cells for glucose oxidation to $\mathrm{CO}_{2}$ through the TCA cycle, interfering with its activity should specifically target cells that resort to aerobic glycolysis to meet their metabolic demands and spare nonproliferative/nonlactate-generating cells (16). 
A

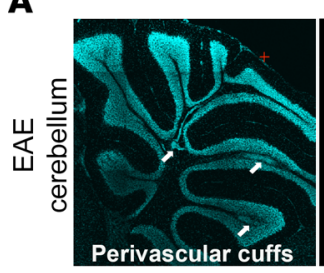

B

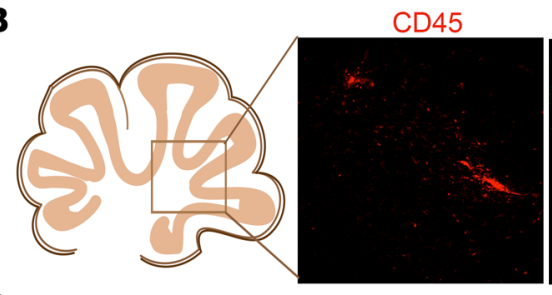

C

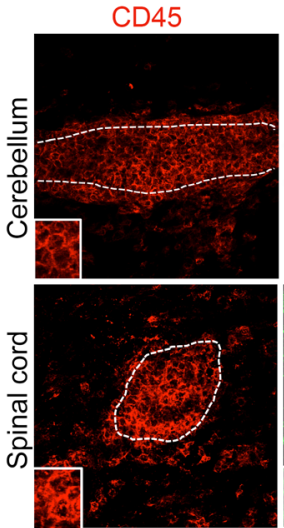

D

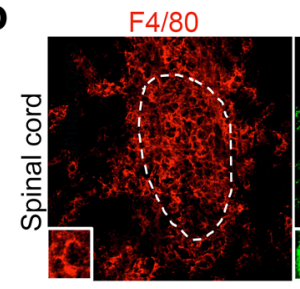

Laminin

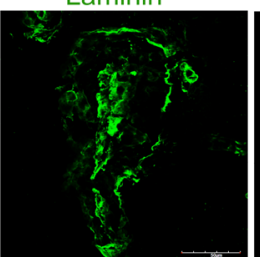

LDHA

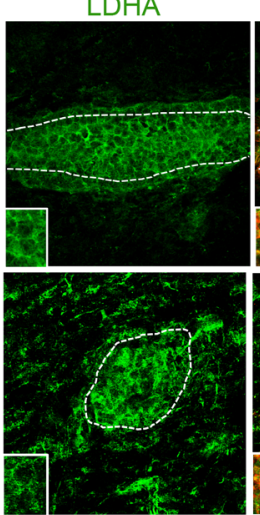

LDHA

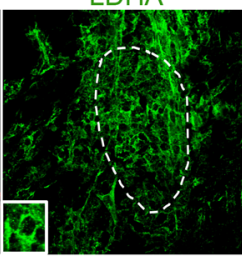

CD45

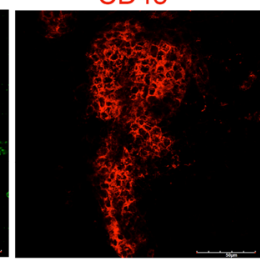

LDHA

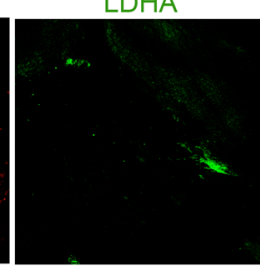

Merged

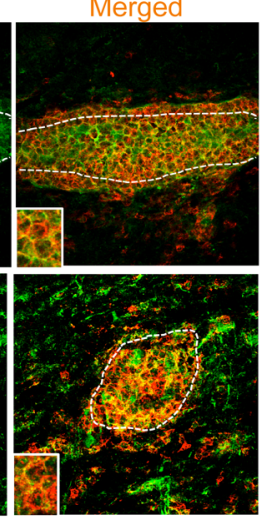

Merged/Hoechst

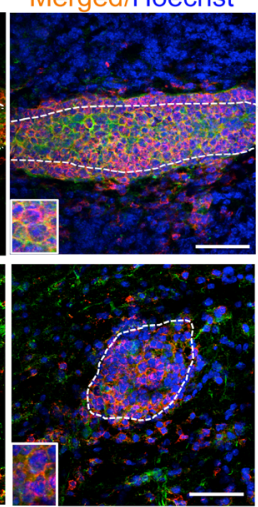

3D reconstruction
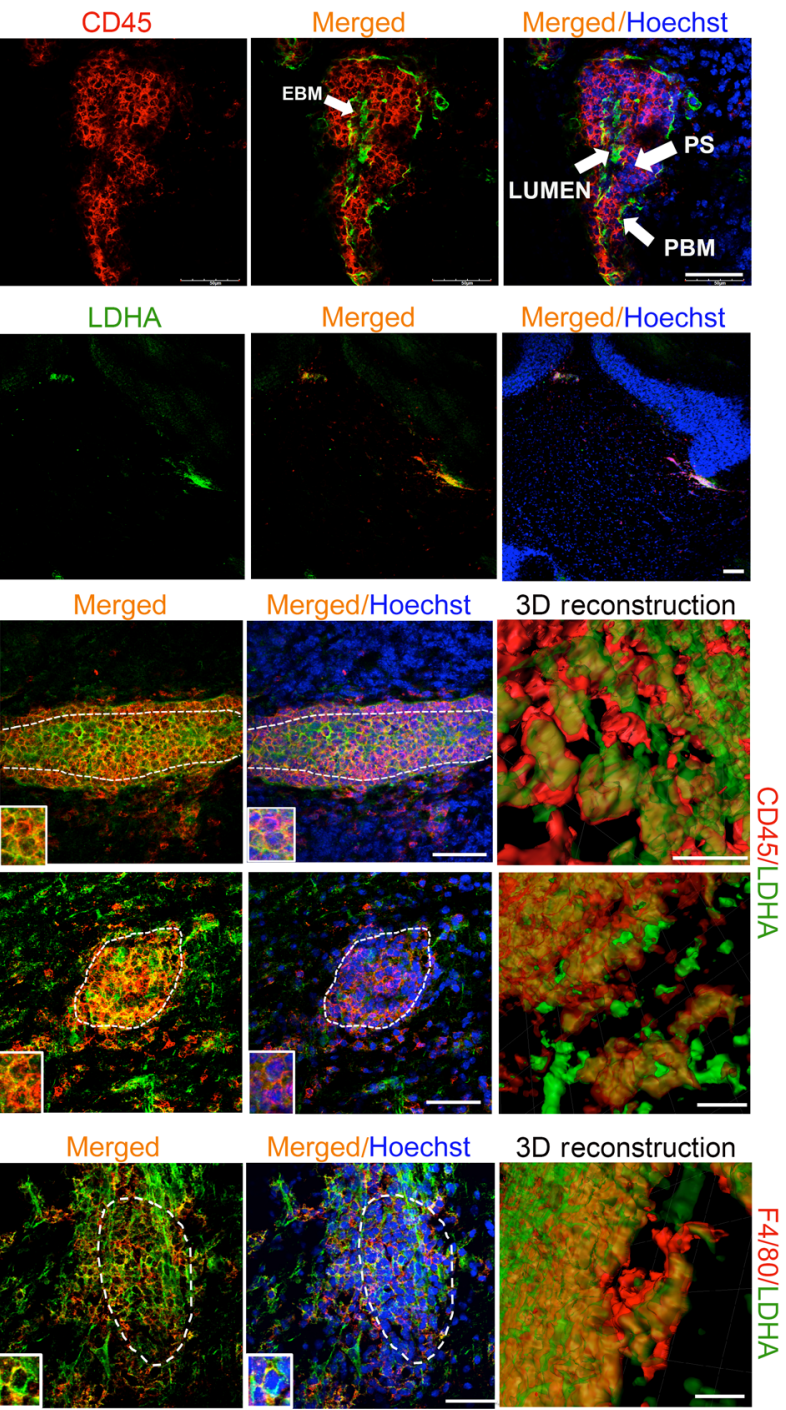

Merged/Hoechst

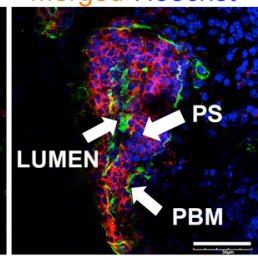

Merged/Hoechst

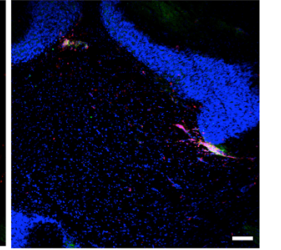

Merged/Hoechst

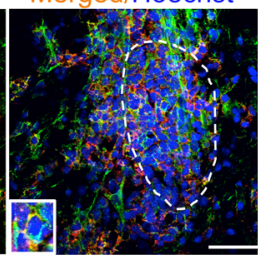

3D reconstruction

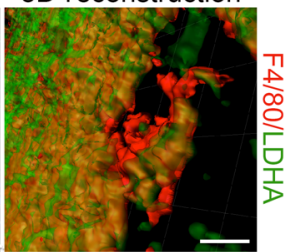

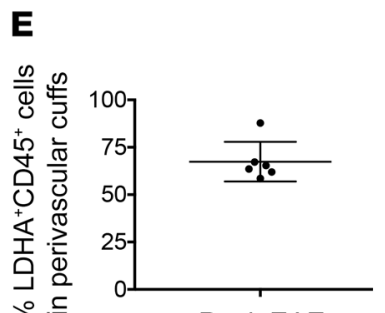

Peak EAE

$\mathbf{F}$

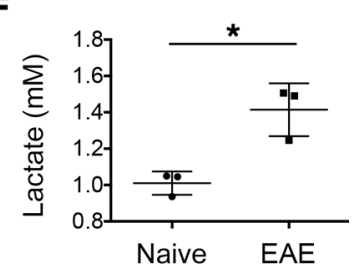

Figure 1. Perivascular cuffs in EAE cerebellum and cervical spinal cord harbor LDHA-expressing leukocytes. (A) Low-magnification image shows a lateral section of an EAE mouse cerebellum (arrows point to perivascular cuffs) along with enlarged images of a perivascular cuff labeled with pan-laminin (green) and the pan-leukocyte marker CD45 (red). Scale bars: $50 \mu \mathrm{m}$. (B) Expression of LDHA and CD45 in a low-magnification cerebellar section of EAE depicts detectable LDHA expression exclusively in CD45+ cells within the white matter. Scale bar: $100 \mu \mathrm{m}$. (C) Representative images of individual cuffs (demarcated by a dotted line) at higher magnification (scale bars: $50 \mu \mathrm{m}$ ) show the expression of LDHA in CD45+ cells in D16 EAE cerebella and spinal cords. Images are representative of 8 mice from 3 independent experiments. 3D reconstruction using Imaris confirmed the presence of LDHA within the CD45+ cells in the inflammatory cuffs (scale bars: $20 \mu \mathrm{m}$ ). (D) Representative images show LDHA expression within F4/80+ macrophages; images are representative of 8 mice from 3 independent experiments (scale bar: $50 \mu \mathrm{m}$ ), with a corresponding 3D reconstruction of this image (scale bar: $20 \mu \mathrm{m}$ ). Insets in $\mathbf{C}$ and $\mathbf{D}$ show magnified cells (original magnification of insets, $\times 120)$. (E) Percentage of LDHA+CD45+ cells within the perivascular cuffs of D16 EAE mice (peak EAE; $n=3$ mice; 2 sections per mouse were analyzed). (F) Lactate levels measured in spinal cord homogenates from 3 D16 EAE mice and 3 naive mice using the L-lactate assay kit. Graphs show the mean \pm SD. Data were compared using a 2-tailed Student's $t$ test. ${ }^{*} P<0.05$.

Monocarboxylate transporters (MCTs) play a crucial role in the sustained production of lactate by transporting excess lactate across cell membranes; MCTs such as MCT-1 and MCT-4, which have a low affinity for lactate, are enriched in cells with high rates of glycolysis $(17,18)$. Studies in cancer cells suggest that MCTs rely on their chaperone extracellular matrix metalloproteinase inducer (EMMPRIN, also known as basignin and CD147) $(19,20)$ for their localization on plasma membranes (21). We have previously described EMMPRIN as an important mediator in the transmigration of leukocytes across the perivascular cuff in MS and its inflammatory model, experimental autoimmune encephalomyelitis (EAE) $(1,2,22)$. Since glycolytic reprogramming favors migration in leukocytes (23), we tested the hypothesis that macrophages rely on EMMPRIN-MCT-4 interaction to meet their metabolic demands in order to cross the BBB into the CNS parenchyma. In particular, we have investigated the relevance of LDHA and the mechanisms of energy utilization in perivascular cuffs in EAE and MS. Moreover, we have evaluated whether perturbation of energy utilization inhibits the transmigration of macrophages, the predominant leukocyte population in perivascular cuffs. Our results 
describe mechanisms of inflammation in EAE and MS and highlight glycolytic macrophages and MCT-4-EMMPRIN interactions that are amenable to perturbation, including by dietary factors, in order to alleviate signs of EAE in mice.

\section{Results}

Macrophages within the perivascular cuffs in EAE mice have high expression of LDHA. In the inflamed postcapillary venules of EAEafflicted mice, leukocytes that have migrated out of the vessellumen across the endothelial cell layer accumulate within the perivascular space between the endothelial and parenchymal basement membranes $(2,3)$; the collective structure is referred to as a perivascular cuff (Figure 1A). Previous studies have emphasized that leukocytes within the perivascular space have remarkable functions, including antigen presentation (6) and secretion of MMPs (24), and these actions probably have high metabolic demands. In support of this, we observed that the expression of LDHA, a key glycolytic enzyme involved in the conversion of pyruvate to lactate, was strongly upregulated in the perivascular cuff on day 16 (D16, a period of prominent clinical disability) in the white matter of the cerebellum, a CNS region where perivascular cuffs are clearly demarcated in EAE (Figure 1B). Higher-magnification micrographs showed that LDHA in perivascular cuffs was expressed by a majority of leukocytes and other cells, probably reactive astrocytes, in the cerebellar white matter (Figure 1C, upper panel) as well as in the spinal cord (Figure 1C, lower panel). We noted that the leukocytes within the parenchyma were largely devoid of LDHA staining, suggesting that these cells may have different metabolic requirements after they have invaded the parenchyma. A 3D rendering using the image reconstruction software Imaris confirmed the expression of LDHA in more than $60 \%$ of $\mathrm{CD} 45^{+}$cells within the perivascular cuffs (Figure 1, C and E). Further, staining with the F4/80 macrophage marker revealed that macrophages within cuffs were highly immune reactive for LDHA (Figure 1D). When we examined lactate, a functional measure of LDHA activity, EAE spinal cord lysates had significantly higher lactate levels compared with levels in control lysates (Figure 1F).

Inflammatory macrophages exhibit stabilization of HIF-1 $\alpha$ (25), a master regulator of LDHA and thus glycolysis. We therefore confirmed the previously published findings on HIF-1 $\alpha$ expression in EAE (26) in the perivascular cuffs of EAE cerebellum and spinal cords (Supplemental Figure 1A; supplemental material available online with this article; https://doi.org/10.1172/JCI124012DS1). The cytoplasmic as well as nuclear expression of HIF-1 $\alpha$ was confirmed with $3 \mathrm{D}$ reconstruction and colocalization using Imaris software (Supplemental Figure 1B). It is worth mentioning that the role of HIF- $1 \alpha$ in mediating glycolytic reprogramming in macrophages is not well understood. Although a recently published study by Le Moan et al. refuted its involvement in driving the EAE disease course (26), mice with knocked out HIF-1 $\alpha$ (23) in myeloid cells showed impaired macrophage migration. This suggests important yet unexplained roles for HIF- $1 \alpha$ in EAE pathogenesis.

In EAE, CNS infiltrates may manifest first as subpial and then as parenchymal perivascular cuffs (5). To study the relevance of LDHA expression in a time-dependent manner, we examined leukocytes during the onset of clinical signs of EAE (D10) and focused on the meninges, since the formation of perivascular cuffs in white matter is not consistent at this time point. We found that more than $50 \%$ of leukocytes expressed LDHA within the meninges on D10 in myelin oligodendrocyte glycoprotein-immunized (MOG-immunized) mice as compared with expression in mice subjected to CFA control, which had fewer leukocytes in the subpial spaces, where only about $10 \%$ of the leukocytes expressed LDHA (Supplemental Figure 2, A and C). Once EAE was established, more than $70 \%$ of leukocytes expressed LDHA in perivascular cuffs, even at a later time point of post-peak disease severity (D21; Supplemental Figure 2, B and D).

LDHA activity is important for the proinflammatory phenotype of macrophages. Proliferation and differentiation are key features of activated leukocytes during inflammation. Since leukocyte proliferation can lead to lactate accumulation in the extracellular compartment (27), we examined the abundance of proliferative leukocytes within cuffs. According to the literature, approximately $20 \%$ of leukocytes proliferate in the subarachnoid space (28) of meninges in EAE-afflicted mice, whereas fewer proliferate within the parenchyma (29). Consistent with these findings, we observed that a modest $10 \%-15 \%$ of $C D 45^{+}$cells were weakly positive for the proliferation marker Ki67 in perivascular cuffs (Supplemental Figure 3A) in comparison with $\mathrm{CD} 45^{+}$leukocytes in meninges, where approximately $25 \%$ to $30 \%$ of these cells had proliferated (Supplemental Figure 3B). Since proliferation is halted in macrophages during inflammation, which implicates HIF-1 $\alpha$-mediated enhancement of glycolysis (30), the nonproliferative macrophages that utilize glycolysis may bear proinflammatory traits.

We tested whether LDHA modulates proinflammatory activities and aids leukocyte infiltration across the BBB. We resorted to macrophages in culture, as these cells typically constitute more than $80 \%$ of leukocytes within perivascular cuffs at peak EAE (24). When bone marrow-derived macrophages (BMDMs) were exposed to a nontoxic $(20 \mu \mathrm{M}$; Figure $2 \mathrm{~A})$ concentration of a small-molecule-specific LDHA inhibitor, 3-dihydroxy-6-methyl7-(phenylmethyl)-4-propylnaphthalene-1-carboxylic acid (FX11) $(12,31)$, we detected a significant decrease in secreted lactate levels as compared with LPS-treated cells (Figure 2B), indicating a decrease in LDHA function. Intracellular lactate accumulation and/or its conversion to pyruvate can regulate glycolysis (11); therefore, when we measured glycolysis by the extracellular acidification rate (ECAR) in FX11-treated, LPS-stimulated cells, we found a significant decrease in both glycolysis (measured by the generation of lactate upon glucose addition) and glycolytic capacity (the maximum capacity of lactate generation upon inhibition of oxidative phosphorylation) in these BMDMs (Figure 2, C and D). Importantly, inhibiting LDHA activity reduced the migratory capacity of macrophages across a Boyden chamber model of the BBB in culture (Figure 2, E and F). Glycolysis feeds anabolic pathways such as the pentose phosphate pathway, which provides necessary precursors for the production of proinflammatory cytokines, and therefore we assessed TNF- $\alpha$ expression upon treatment with FX11. We found a significant decrease in the production of TNF- $\alpha$ upon FX11 treatment in LPS-activated cells (Figure 2G). Further, we tested whether the inhibitory effects of FX11 on macrophage migration were mediated by chemotactic signals. Indeed, FX11 decreased the production of MCP-1 (also known as CCL2) and IP-10 (also known as CXCL10) chemokines in macrophages 
A

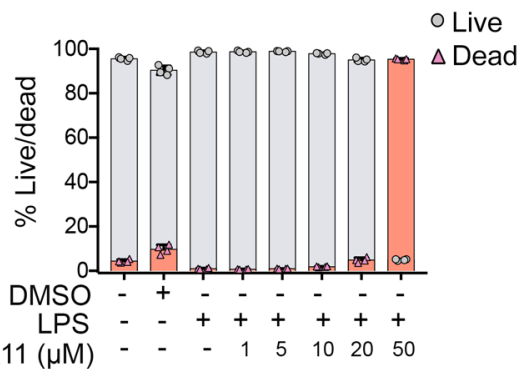

B

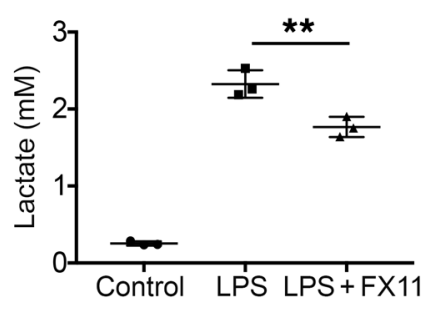

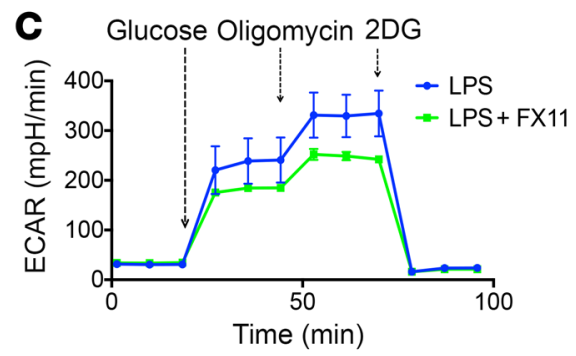

D

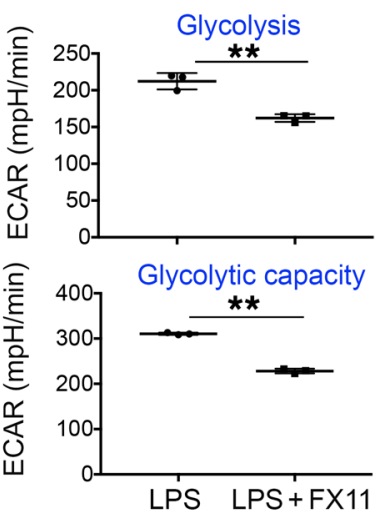

E

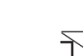
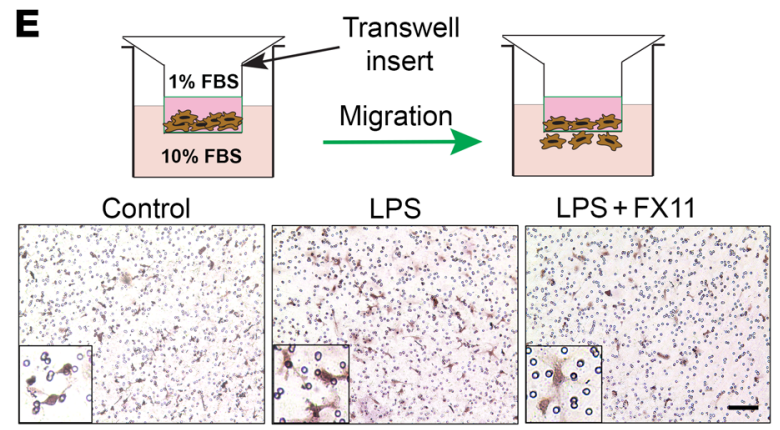

$\mathbf{F}$

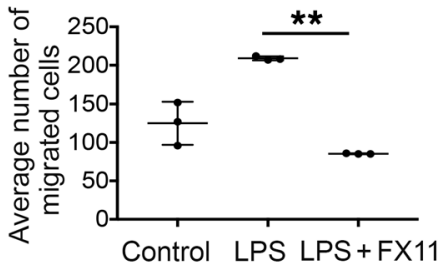

G

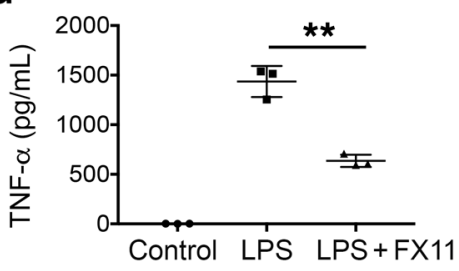

H

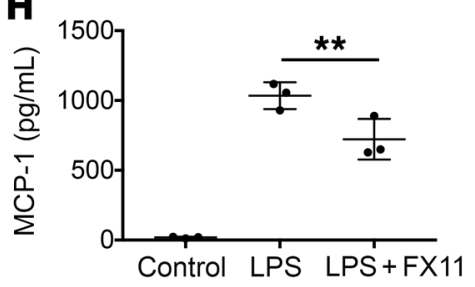

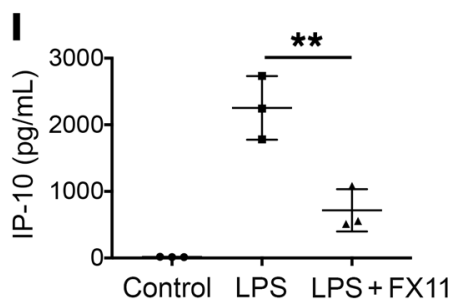

Figure 2. Glycolysis drives proinflammatory activities of macrophages. (A) Live/dead assay as measured by calcein AM (live cells; gray) and PI (dead cells; red) staining of LPS-stimulated BMDMs in the presence of FX11. Results are representative of 3 independent experiments run in quadruplicate. (B) Graph represents lactate levels in LPS-stimulated or untreated cells in the presence or absence of FX11. (C) ECAR measurement in FX11-treated and untreated inflammatory BMDMs. Results are representative of 2 independent experiments run in quadruplicate; values were normalized to micrograms of protein. (D) Graphs depict glycolysis (mean ECAR upon addition of glucose) and glycolytic capacity (mean ECAR upon addition of oligomycin). Values were normalized to micrograms of protein $(n=4)$. (E) The effects of FX11 on macrophage transmigration were studied by plating the BMDMs in Transwell inserts (Boyden chambers) in the presence of medium with 1\% FBS in the upper chamber and 10\% FBS medium in the lower chamber (schematic). Representative images of the transmigrated cells are shown. Scale bar: $50 \mu \mathrm{m}$ (original magnification, $\times 30$ for insets). The assay was performed in quadruplicate, and the results are representative of 2 independent experiments. (F) Graph represents the average number of cells that migrated over an 8-hour period in the presence or absence of FX11. (G) TNF- $\alpha$ ELISA and (H) Luminex analysis of chemokines revealed a significant reduction in MCP-1 (also known as CCL2) and (I) IP-10 (also known as CXCL10) upon treatment with FX11 in LPS-stimulated cells. All graphs show the mean \pm SD ( $n=3$ unless otherwise indicated). Means were compared using a 2-tailed Student's $t$ test (D) or 1-way ANOVA with Tukey's post hoc test (for multiple groups in B, F, and $\mathbf{G}-\mathbf{I}) .{ }^{* *} P<0.01$.

(Figure 2, H and I). These results support the importance of LDHA activity in macrophages that drives their proinflammatory activities including infiltration into the CNS.

Perivascular cuffs in EAE are laden with macrophages that express MCT-4, an exporter of lactate. Accumulation of lactate within a cell could alter glycolysis by a possible feedback inhibition mechanism (11), so it would be important to pump out excess lactate while maintaining intracellular lactate production. MCT- 1 and MCT-4 are transporters that secrete lactate (32), and MCT- 4 is a particularly important exporter of lactate in glycolytic cells $(32,33)$. Thus, we evaluated D16 CNS samples and found that within the white matter, expression of MCT- 4 was localized to the perivascular cuff region, where it was found to be prominently expressed in a majority of $\mathrm{CD} 45^{+}$cells in both the cerebella and spinal cords of EAE mice (Figure 3, A, B, and D). As with LDHA, we observed that several leukocytes in the parenchyma did not have detectable MCT4 expression, suggesting a switch in metabolic programming in these cells upon infiltration into the parenchyma. A 3D Imaris rendering confirmed the expression of MCT- 4 on CD $45^{+}$leukocytes including in $\mathrm{F} 4 / 80^{+}$macrophages (Figure $3, \mathrm{~B}$ and $\mathrm{C}$ ).

As seen with LDHA, MCT-4 was expressed in meningeal leukocytes as early as D10 (Supplemental Figure 4A), with more than $50 \%$ of the leukocytes found positive for MCT- 4 as compared with CFA control leukocytes (Supplemental Figure 4C). Similarly, more than $60 \%$ of leukocytes expressed MCT- 4 in post-peak D21 perivascular cuffs (Supplemental Figure 4B), suggesting that these cells express this lactate transporter even at later stages of the disease. Notably, we also detected MCT-4 expression in reac- 
A

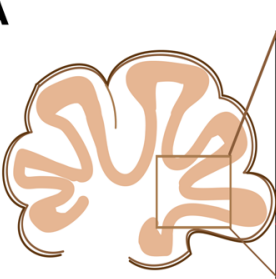

B
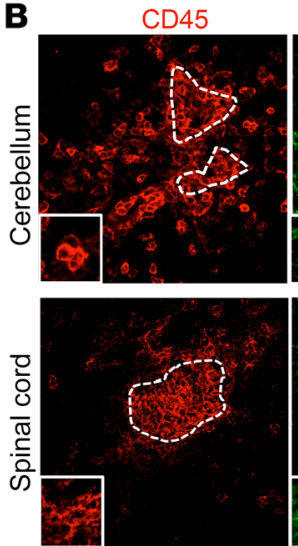

C

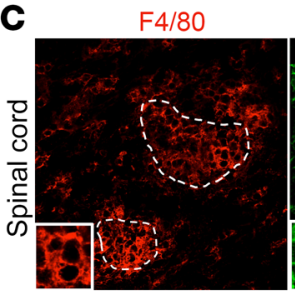

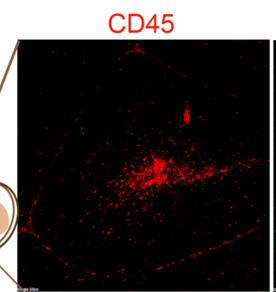

MCT-4
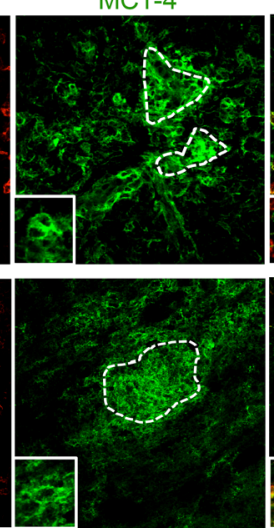

MCT-4

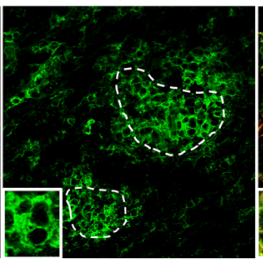

MCT-4

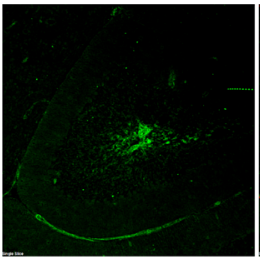

Merged
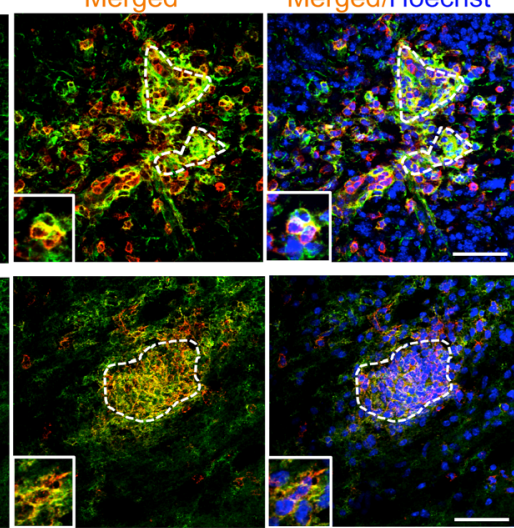

Merged

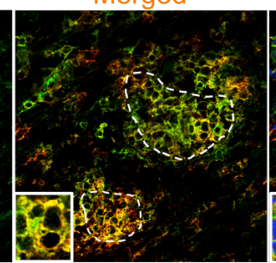

Merged/Hoechst

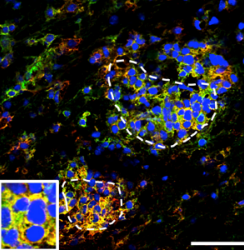

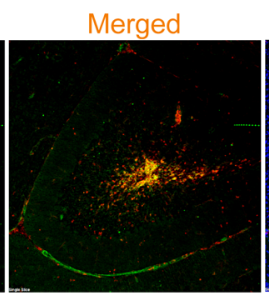

Merged/Hoechst

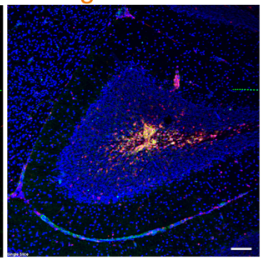

3D reconstruction
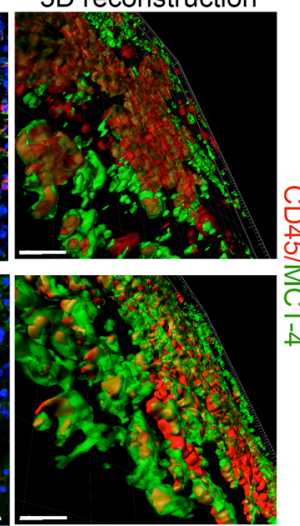

3D reconstruction

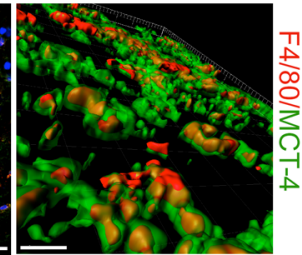

D

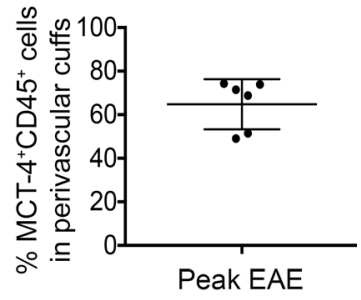

Figure 3. Leukocytes exiting the perivascular cuffs in D16 EAE express MCT-4, a key transporter of lactate in glycolytic cells. (A) Low-magnification image of MCT-4 staining shows its expression restricted to the cells within and in the proximity of cuffs in D16 EAE cerebellum. Scale bar: $100 \mu \mathrm{m}$. (B) High-magnification micrographs of perivascular cuffs in cerebellum and spinal cord highlight the expression of MCT-4 within CD45 ${ }^{+}$cells; images are representative of 8 mice from 3 independent experiments. Scale bar: $50 \mu \mathrm{m}$. Insets show magnified cells (original magnification, x120). 3D reconstructed images confirm the expression of MCT-4 within CD45+ leukocytes (scale bars: $20 \mu \mathrm{m}$ ). (C) Representative images of the spinal cord of a D16 EAE mouse depicting F4/80+ macrophages with prominent levels of MCT-4. Scale bar: $50 \mu \mathrm{m}$. Images are representative of 6 mice from 3 independent experiments. 3D reconstructed image confirmed the expression of F4/80+ macrophages (scale bar: $10 \mu \mathrm{m}$ ). (D) Graph shows the percentage of MCT-4+ leukocytes in perivascular cuffs in D16 EAE mice ( $n=3$ mice; 2 sections per mouse were analyzed).

tive astrocytes abutting the perivascular cuffs in the EAE cerebellum (Supplemental Figure 5A). For a thorough analysis, we also assessed the expression of MCT- 1 in CD $45^{+}$leukocytes and reactive astrocytes in perivascular cuffs, which suggested the presence of MCT-1 in astrocytes as well as in a small subset of leukocytes (Supplemental Figure 5B).

In order to corroborate the significance of LDHA and establish a role for MCT-4 in aiding proinflammatory activities in BMDMs, we performed siRNA-mediated knockdown of LDHA and MCT-4 in BMDMs (Figure 4, A and C). Notably, knockdown of MCT- 4 and, expectedly, LDHA, resulted in decreased LDHA expression in BMDMs treated with LPS (Figure 4, B, D, and E). Further, knockdown of LDHA and MCT-4 significantly reduced lactate levels in LPS-treated BMDM supernatant (Figure 4F). We then sought to study the relevance of LDHA and MCT-4 in mediating proinflammatory activities in knockdown cells (Figure 4G). We found a modest yet significant reduction in TNF- $\alpha$ production after 6 hours of LPS treatment in LDHA- and MCT-4-knockdown cells (Figure 4G). However, this reduction was not observed after
24 hours of stimulation (data not shown), possibly due to a lack of a complete reduction of either gene. Also, the transmigration assay confirmed that migration of LDHA- and MCT-4-knockdown BMDMs was significantly reduced when compared with scRNA-transfected cells in response to LPS treatment (Figure $4 \mathrm{H}$ ).

EMMPRIN, a molecular chaperone for MCT-4, governs proinflammatory functions in macrophages. We addressed the mechanisms by which MCT-4 is regulated in leukocytes; specifically, we evaluated EMMPRIN, as it is known to chaperone MCTs in cancer cells (21). We found EMMPRIN to be strongly associated with MCT-4 in cells within perivascular cuffs (Figure 5A). Western blot analysis of EMMPRIN in EAE spinal cords revealed elevated expression of various glycosylated forms of this protein, ranging from $40 \mathrm{kDa}$ to more than $80 \mathrm{kDa}$ (Figure 5B). To further assess EMMPRIN-MCT4 interactions in both inflammatory and noninflammatory conditions, we treated macrophages with LPS and found that MCT-4 increased within 24 and 48 hours of treatment (Supplemental Figure 6A). Using co-IP with MCT-4 antibody to "pull" for interacting partners, we found that an approximately $50-\mathrm{kDa}$ form of EMM- 
A

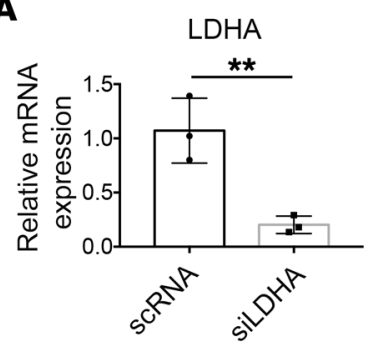

C

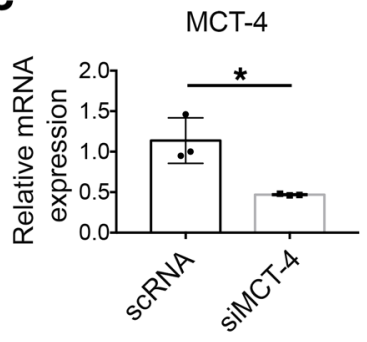

G

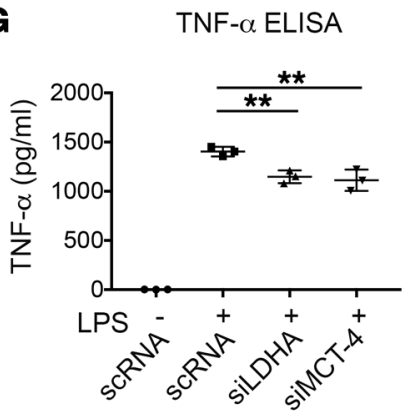

B

\section{D}
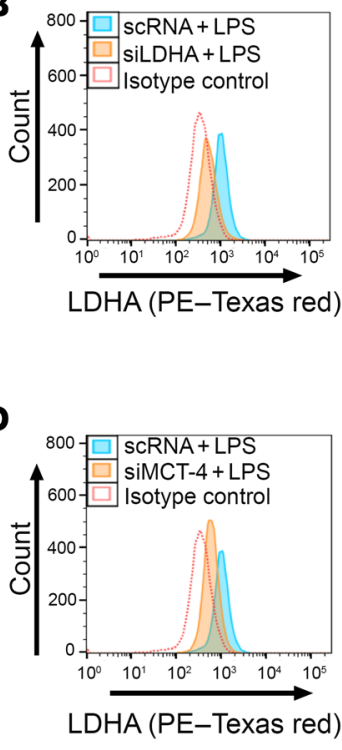

$\mathbf{F}$
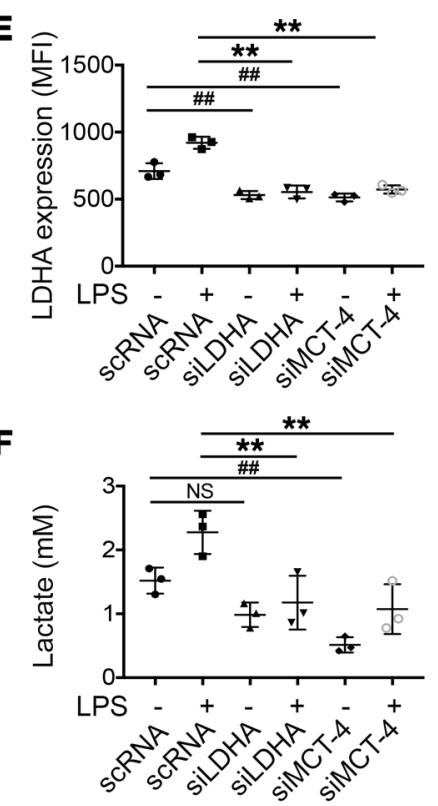

Figure 4. siRNA-mediated knockdown of LDHA and MCT-4 dampens inflammation in BMDMs. (A) RT-PCR of LDHA transcripts in scRNA- and LDHA siRNA-transfected BMDMs. (B) Flow plot shows the MFI of LDHA (PE-Texas red) in different conditions. (C) MCT-4-knockdown experiments detected reduced MCT-4 transcript levels by RT-PCR. (D) Flow plot shows LDHA MFI in MCT-4-knockdown cells. (E) Graph shows quantification of LDHA MFI in LDHA- and MCT-4-knockdown cells from the flow cytometric plots shown in B and D. (F) Supernatants analyzed for lactate revealed a significant reduction in lactate secretion in LDHA- and MCT-4knockdown cells from LPS-stimulated BMDMs. (G) TNF- $\alpha$ ELISA and (H) transmigration assay of BMDMs subjected to knockdown of LDHA and MCT-4. All assays were carried out in triplicate or quadruplicate and are representative of at least 2 independent experiments. All graphs show the mean $\pm \mathrm{SD}$. Means were compared using 1-way ANOVA with Tukey's post hoc test. ${ }^{*} P<0.05$ and ${ }^{*} P$ $<0.01 ;{ }^{\# \#} P<0.05$ versus scRNA-only condition.

PRIN interacted with MCT-4 in LPS-treated as well as untreated macrophages (Figure $5 \mathrm{C}$ ), indicating that the 2 proteins interact in macrophages during both their resting and activated states.

To further our understanding of the functions of EMMPRIN, we performed siRNA-mediated knockdown of EMMPRIN in macrophages and isolated their membrane fractions. Upon knockdown of EMMPRIN (Figure 5D), MCT-4 expression on membranes was significantly reduced (Figure $5 \mathrm{E}$ ). This was also supported by immunofluorescence staining of MCT-4 on BMDMs, which primarily localized on the plasma membrane, suggesting that knockdown of EMMPRIN as well as LDHA and MCT-4 significantly reduced membrane MCT-4 expression levels in BMDMs (Supplemental Figure 6B). These results suggest that EMMPRIN regulates the expression of MCT- 4 in BMDMs by chaperoning it to macrophage membranes. Relevant to this observation, we found that an LPS-mediated increase in glycolysis and glycolytic capacity was significantly abrogated in EMMPRIN-knockdown cells (Figure 6A), as was also confirmed by decreased lactate secretion from LPS-stimulated EMMPRIN-knockdown cells (Figure 6B). In further support of this finding, we observed that EMMPRIN-expressing cells in perivascular cuffs of the spinal cords of EAE mice also expressed LDHA (Supplemental Figure 7A) and that LPS stimulation of macrophages in cultures increased the number of $\mathrm{EMMPRIN}^{+} \mathrm{LDHA}^{+}$cells (Supplemental Figure 7B). Importantly, knockdown of EMMPRIN significantly reduced LDHA expression in inflammatory macrophages (Figure 6C), leading to a significant reduction in the production of TNF- $\alpha$ (Figure 6D). Furthermore, similar to LDHA and MCT-4 knockdown cells, the migration of EMMPRIN-knockdown BMDMs was significantly retarded across the Boyden chamber (Figure 6E). These results highlight the critical role of the EMMPRIN-MCT-4 interaction in lactate metabolism during inflammation.

Inhibition of EMMPRIN-MCT-4 interaction reduces macrophage activation. In order to further interrogate the relevance of MCT-4 and its interaction with EMMPRIN in macrophages, we used $\alpha$-cyano-4-hydroxy-cinnamic acid (CHCA), a reversible and relatively specific inhibitor of MCT-4, albeit through unknown mechanisms (33, 34). We determined that CHCA was not toxic to macrophages, even at $1 \mathrm{mM}$ concentration (Figure 7A). Notably, $400 \mu \mathrm{M}$ CHCA significantly reduced the secretion of lactate in activated macrophages (Figure 7B). Further, CHCA-treated macrophages had a reduced ECAR (Figure 7C), in which both the glycolysis and glycolytic capacity of BMDMs were reduced (Figure 7, D and E). These observations were similar to the effects observed with either EMMPRIN knockdown or FX11-mediated LDHA inhibition described earlier.

It is known that knockdown of MCT-4 reduces glycolytic enzymes in macrophages (34). Also, as described earlier, siRNAmediated knockdown of MCT-4 decreased the expression of LDHA and secretion of lactate in BMDMs (Figure 4, E and F). In this regard, we observed that LPS-induced expression of EMMPRIN, LDHA, and HIF- $1 \alpha$ in BMDMs was reduced in the presence of CHCA (Figure 7F), possibly due to a feedback inhibition by accumulation of lactate intracellularly. Since no changes were observed in MCT-4 interaction with 
A
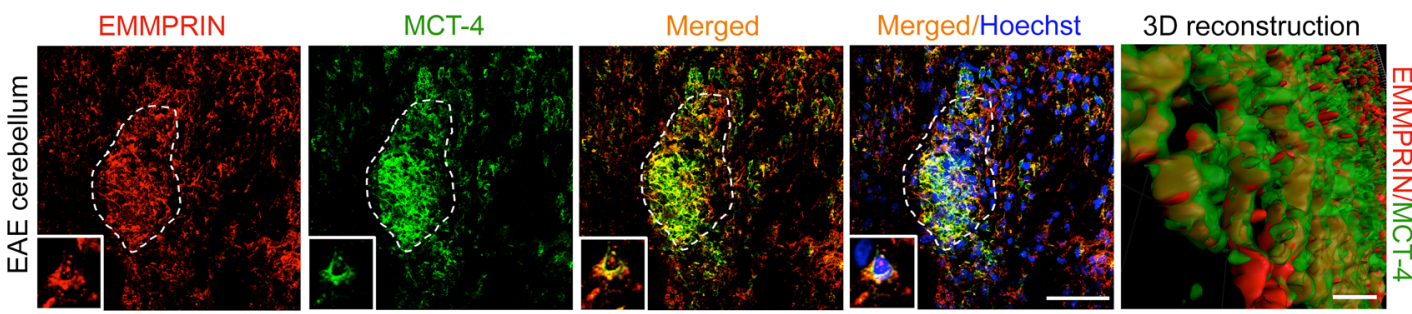

B

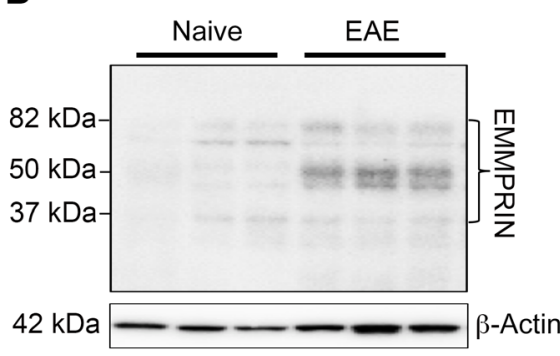

C

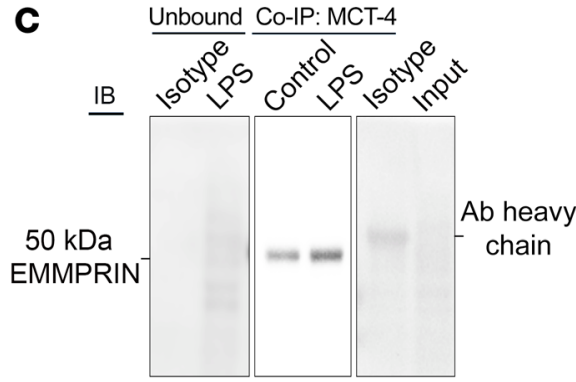

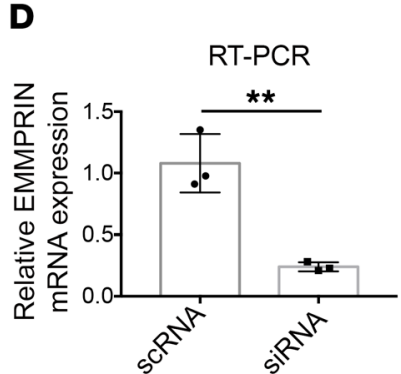

$\mathbf{E}$
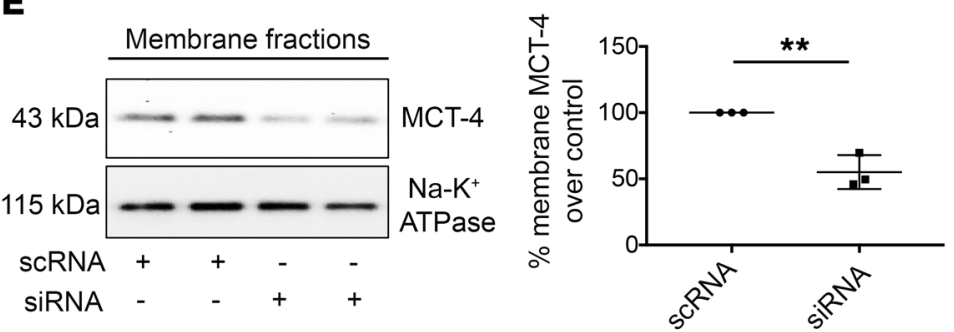

Figure 5. MCT-4 requires its chaperone, the extracellular MMP inducer EMMPRIN, in macrophages. (A) Micrographs of MCT-4+ $4^{+}$cells (green) with expression of EMMPRIN (red) in a perivascular cuff (scale bar: $50 \mu \mathrm{m}$ ); insets show magnified cells (original magnification, $\times 200$ ). 3D reconstruction image confirmed the overlap between MCT-4 and EMMPRIN channels (scale bar: $10 \mu \mathrm{m}$ ). Image are representative of 4 mice. (B) Immunoblot (IB) with upregulation of multiple glycosylated forms of EMMPRIN in D16 EAE spinal cords as compared with naive controls. $n=3$ mice per group analyzed. (C) IB confirmed the interaction between a 50-kDa glycosylated form of EMMPRIN upon co-IP with MCT-4 as well as in unbound fractions. $n=2$ independent experiments; protein was pooled from duplicate treatments. (D) Relative transcript levels (RT-PCR, comparative $\triangle \Delta C$ t method) show siRNA-mediated knockdown of EMMPRIN in transfected BMDMs. (E) IB for MCT-4 and loading control Na-K+ ATPase in the membrane fractions of EMMPRIN-knockdown BMDMs shows reduced levels of MCT-4 in the knockdown cells. All assays were performed in triplicate or quadruplicate for 3 independent experiments unless otherwise indicated. All graphs show the mean \pm SD. Means were compared using a 2-tailed Student's $t$ test. ${ }^{* *} P<0.01$.

the 50-kDa EMMPRIN in untreated and LPS-treated macrophages (Figure 5C), we studied the higher glycosylated forms of EMMPRIN, which govern proinflammatory functions of macrophages (35). Using a different EMMPRIN antibody, we found that, whereas the $42-\mathrm{kDa}$ EMMPRIN form did not interact with MCT-4, a higher glycosylated EMMPRIN ( $50-56 \mathrm{kDa})$ associated with MCT-4 (Figure 7G). However, no significant differences were observed in response to either LPS or CHCA conditions in the approximately 50-kDa EMMPRIN form. Nonetheless, another glycosylated form of EMMPRIN (>100 $\mathrm{kDa}$ ) increased upon LPS activation and was reduced with CHCA treatment (Figure 7, G and $\mathrm{H}$ ). These results suggest that high-molecular-weight glycosylated forms of EMMPRIN may play a crucial role in guiding MCT-4 to macrophage membranes. These findings also show that functional MCT-4 inhibition reduced EMMPRIN-MCT-4 interaction, which could also be a reflection of decreased EMMPRIN expression upon treatment with CHCA.

Finally, we studied the consequence of pharmacological perturbation of MCT- 4 function by evaluating the transmigration of macrophages in the Boyden chamber model of the BBB. We found that the elevated transmigration of macrophages upon LPS activation was significantly retarded by CHCA treatment (Figure 7, I and J), as was observed with MCT- 4 knockdown in BMDMs (Figure $4 \mathrm{H}$ ), further supporting the hypothesis that an intact lactate machinery, regulated by EMMPRIN-MCT-4 interaction, is crucial for inflammatory activities of macrophages.

CHCA treatment decreases disease severity in EAE mice. Mice immunized for EAE were injected i.p. daily with $25 \mathrm{mg} / \mathrm{kg}$ (data not shown) or $50 \mathrm{mg} / \mathrm{kg}$ CHCA from D4 after immunization (Figure $8 \mathrm{~A}$ ) while asymptomatic. We noted that the weight drop in EAE mice during severe clinical disability (D14-D20) was prevented by CHCA (data not shown). While mice in the vehicle- and CHCA-treated groups succumbed at comparable rates to EAE between D10 and D12, the subsequent clinical disability was dampened by CHCA compared with vehicle (Figure 8B). When vehicle-treated mice had an average maximum score of 2.5 on D12 (limp tail and paresis of hind limbs and forelimbs) on a 5-point scale, CHCA-treated mice had a score of 1 (limp tail only).

We analyzed the formation of cuffs in spinal cords on D16 and found a significant reduction in the number of cuffs in CHCA-treated mice as compared with vehicle-treated animals 

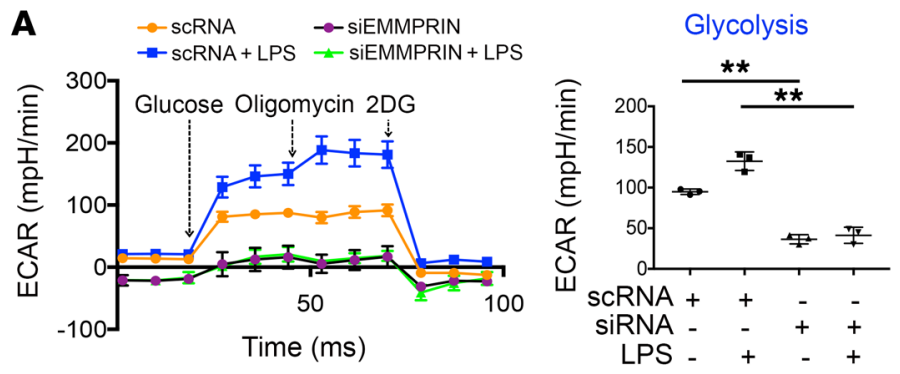
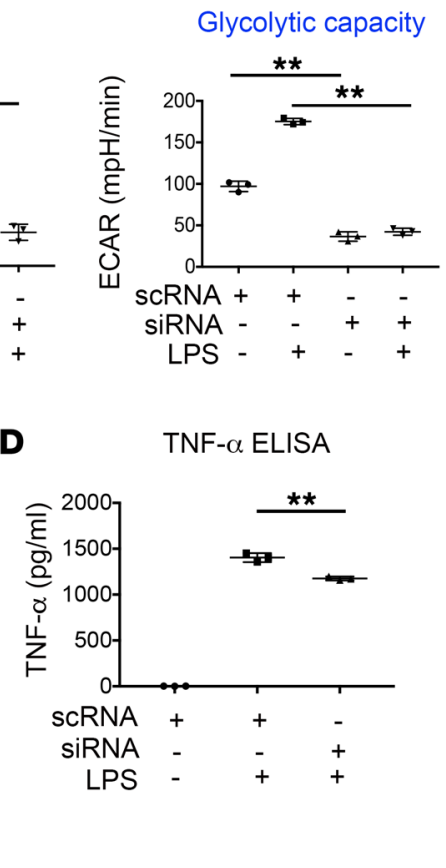

B
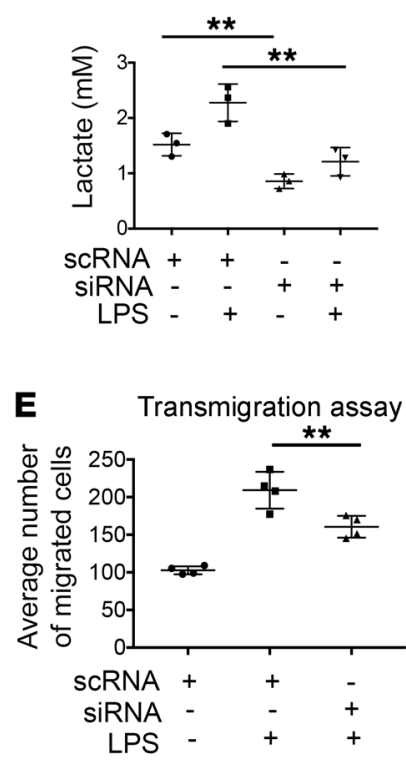
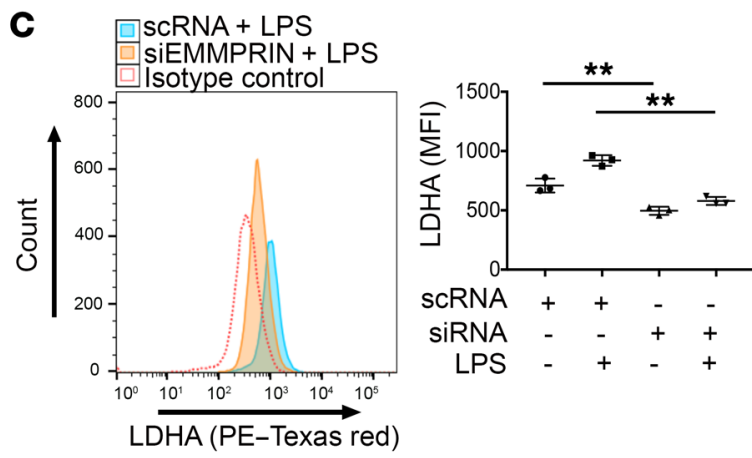

Figure 6. EMMPRIN knockdown results in decreased glycolysis and reduced proinflammatory functions in macrophages. (A) ECAR measurement for cells transfected with EMMPRIN siRNA. Graphs depict glycolysis and glycolytic capacity in EMMPRIN-knockdown cells. Final values were normalized to micrograms of protein $(n=3)$. (B) Graph depicts lactate levels in supernatants from EMMPRIN-knockdown cells either in the presence or absence of LPS. (C) Flow plot and representative graph show LDHA MFI in EMMPRIN-knockdown cells. (D) TNF- $\alpha$ ELISA and (E) transmigration assay suggested a relevance for EMMPRIN in regulating these aspects of BMDM inflammation. All assays were performed in triplicate or quadruplicate for 3 independent experiments unless otherwise indicated. All graphs show the mean \pm SD. Means were compared by 1-way ANOVA with Tukey's post hoc test. ${ }^{* *} P<0.01$.

(Figure 8C). Using Imaris software, the perivascular cuffs were rendered a surface (green), while the distance of each infiltrate (spheres) from this surface was color coded, with purple being the closest and red being the furthest from the cuff. The distance transformation of these cuffs (Figure 8D) showed that the infiltrating leukocytes in vehicle-treated EAE mice traveled from cuffs deep into the parenchyma (we set the cutoff at $100 \mu \mathrm{m}$, chosen to exclude cells from other nearby cuffs) (Figure 8E). In contrast, CHCA kept a majority of cells in proximity (within 20 $\mu \mathrm{m}$ ) of cuffs (Figure 8F), corroborating their inhibited migration. Also, immunofluorescence (CD45 and laminin) and histological (H\&E) analyses on D16 show that the CHCA-treated spinal cords had less meningeal infiltration than was detected in the spinal cords of vehicle-treated EAE mice (Figure 8, G and H), which was validated by blinded rank order analysis (Figure 8I; based on $H \& E$ staining). Since we saw a decrease in inflammation upon CHCA treatment in EAE mice, we examined CD11b ${ }^{+}$Ly6G monocytes in the circulation (D10) prior to their appearance at perivascular cuffs and found that their numbers were significantly higher in the periphery of CHCA-treated mice (Figure 8, J and $\mathrm{K})$, indicating impediment of their migration to the CNS. Further, we examined whether CHCA treatment altered the levels of LDHA on peripheral leukocytes and found a significant reduction in the number and level of $\mathrm{LDHA}^{+} \mathrm{CD} 45^{+}$cells (Figure 8, $\mathrm{L}-\mathrm{N}$ ) upon CHCA treatment in EAE mice. Since inhibition of LDHA reduced the expression of IP-10 in macrophages (Figure 2I), a key $\mathrm{T}$ cell chemokine, we sought to determine whether infiltration of both $\mathrm{F} 4 / 80^{+}$myeloid cells and $\mathrm{CD} 3^{+} \mathrm{T}$ cells in CHCA-treated spinal cords is affected (Supplemental Figure 8). We found that infiltration of both $\mathrm{CD}^{+} \mathrm{T}$ cells and $\mathrm{F} 4 / 80^{+}$myeloid cells was significantly reduced in CHCA-treated spinal cords compared with vehicle-treated controls (Supplemental Figure 8, A and B), suggesting that CHCA, either directly or indirectly via its effect on chemokine production by macrophages, may affect $\mathrm{T}$ cell migration into EAE spinal cords. Further, when we analyzed the levels of LDHA on either CD $45^{+} \mathrm{Ly}_{6 \mathrm{G}}{ }^{-} \mathrm{CD} 11 \mathrm{~b}^{+}$monocytes (Supplemental Figure 8C) or $\mathrm{CD}^{+} \mathrm{T}$ cells (Supplemental Figure 8E) in blood from D16 EAE mice, we found that the expression of LDHA was significantly elevated on monocytes (Supplemental Figure 8D), but not on T cells (Supplemental Figure 8F), when compared with the naive condition.

To assess the direct effects of CHCA on T cells, we treated either the polyclonally activated splenocytes (Supplemental Figure 9, A-D) or the Th1 (Supplemental Figure 9, E and F) or Th17polarized cells (Supplemental Figure 9, G-H) in vitro. We found that CHCA did not affect the proliferation of splenocytes (Supplemental Figure 9B). However, it significantly decreased IFN- $\gamma^{+} \mathrm{T}^{-}$bet $^{+}$ Th1 cells in splenocytes (Supplemental Figure 9C), but increased the Th1 polarization in pure T cell cultures (Supplemental Figure 9, $\mathrm{E}$ and F), without affecting the polarization of Th17 cells in either polarization paradigm (Supplemental Figure 9, D, G, and H). This further reinforces the observation that the presence of other modulatory cells, probably monocytes in splenocytes, may significantly affect pathogenic Th1-polarized cells in the presence of CHCA in vivo. However, further experiments are needed to assess the effects of $\mathrm{CHCA}$ on $\mathrm{T}$ cell polarization in vivo. 
A

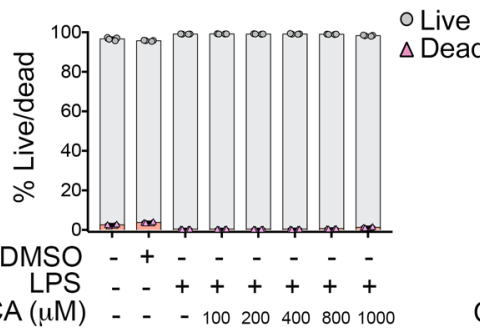

D

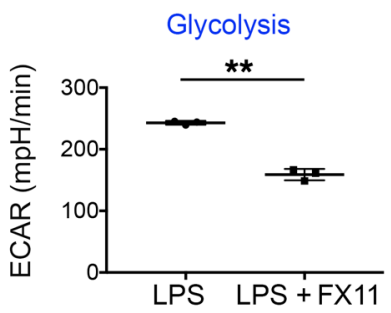

B

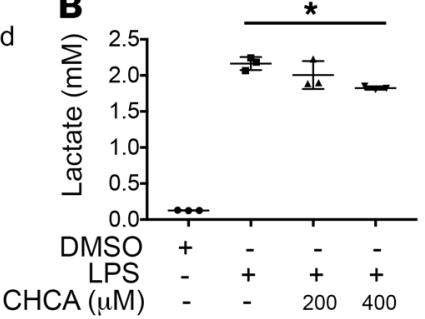

E

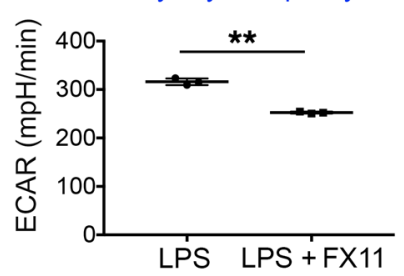

C

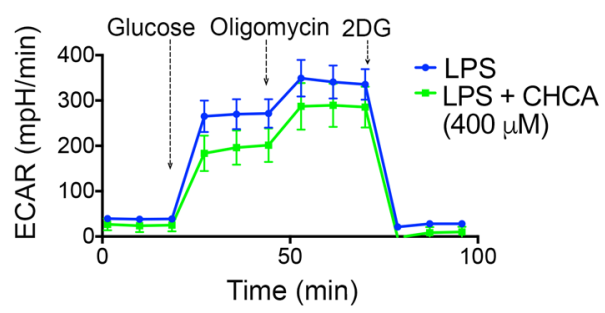

$\mathbf{F}$

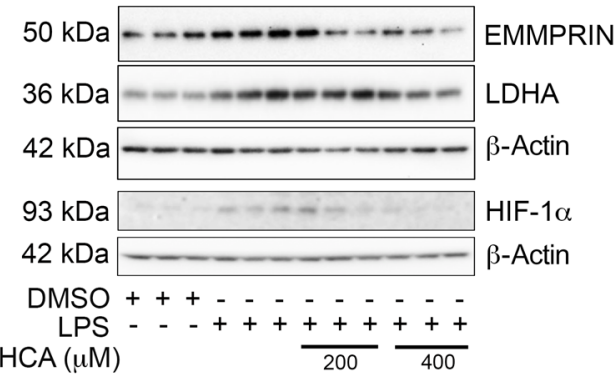

G

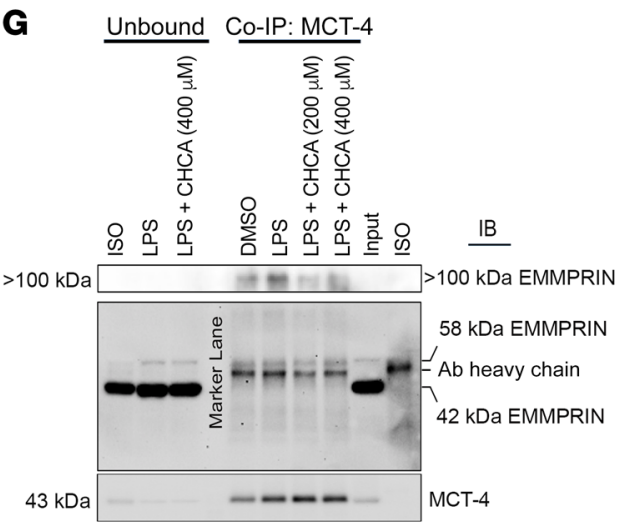

H
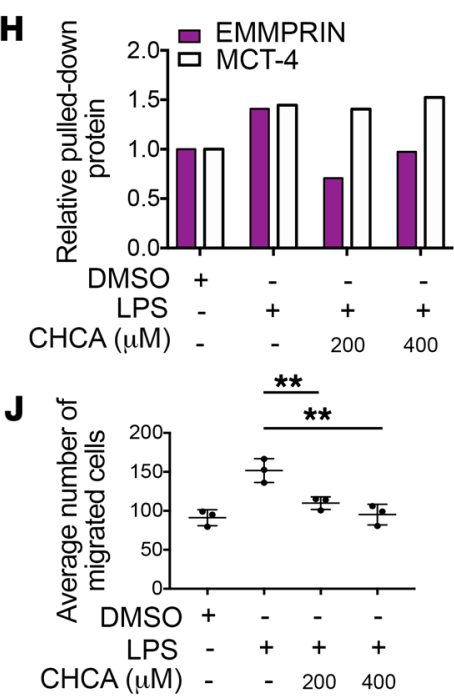

I Control LPS

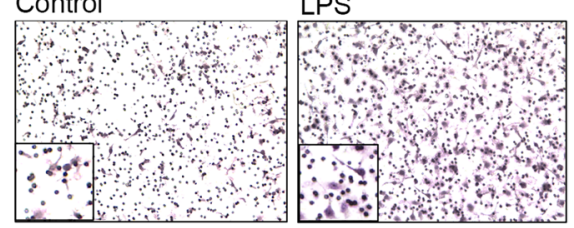

$\mathrm{LPS}+\mathrm{CHCA}(200 \mu \mathrm{M}) \mathrm{LPS}+\mathrm{CHCA}(400 \mu \mathrm{M})$

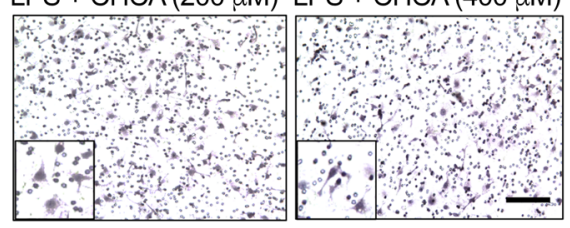

Figure 7. MCT-4 inhibition decreases glycolysis in BMDMs and dampens their proinflammatory activity. (A) Live/dead assay as measured by calcein-AM (live cells; gray) and PI (dead cells; red) staining in macrophages in different conditions. (B) Lactate levels in supernatants of LPS-stimulated BMDMs in the presence or absence of 200 and $400 \mu$ M CHCA. (C) ECAR measurements of LPS-stimulated cells treated with $400 \mu$ M CHCA. Results are representative of 2 independent experiments run in quadruplicate. (D and E) Graphs depict (D) glycolysis and (E) glycolytic capacity in LPS and LPS plus CHCA conditions. Values were normalized to micrograms of protein. Means compared with 2-tailed Student's $t$ test. (F) IB of LPS-stimulated BMDMs shows a reduction in EMMPRIN, LDHA, and HIF-1 $\alpha$ within 12 hours of CHCA treatment. (G) IB shows different glycosylated forms of EMMPRIN when lysates from CHCA-treated inflammatory BMDMs were "pulled" with MCT-4 antibody (co-IP: MCT-4). The MCT-4 band is shown for specificity of the co-IP antibody. (H) Graph represents the relative change in protein levels of MCT-4 and EMMPRIN (>100 kDa; high glycosylated or multimeric form) in co-IP conditions upon CHCA treatment in LPS-stimulated BMDMs. Graph represents 2 experiments, with protein pooled from triplicate treatments each. (I) Representative bright-field images of BMDMs transmigrated in LPS and LPS plus CHCA conditions in a Boyden chamber setup. Scale bar: $50 \mu \mathrm{m}$ (original magnification, $\times 20$ for insets). (J) Graph represents the average number of cells that migrated across the chamber in different conditions. Results are representative of 2 independent experiments run in quadruplicate. Graphs show the mean \pm SD. Means were compared using 1-way ANOVA with Tukey's post hoc test unless otherwise indicated. ${ }^{*} P<0.05$ and ${ }^{* *} P<0.01$.

Inflammatory lesions within MS brains harbor leukocytes with glycolytic traits. We assessed the relevance of the EAE results by examining autopsied brains from deceased subjects with MS. At the sites of perivascular cuffs (Figure 9A) containing $\mathrm{CD}^{+} 8^{+}$macrophages (Figure 9) or $\mathrm{CD}^{4} 5^{+}$leukocytes (Supplemental Figure 10), we could clearly detect LDHA, MCT-4, and HIF-1 $\alpha$, corresponding with CD68 ${ }^{+}$macrophages (Figure 9, B-D) or CD45 leukocytes (Supplemental Figures 10 and 11). EMMPRIN-expressing cells were immunoreactive for MCT- 4 in perivascular cuffs (Figure 9E), corroborat- ing the EAE data indicating the important role of EMMPRIN in the maintenance of MCT-4 levels in infiltrating leukocytes. In order to assess whether the age of the MS subjects determined the glycolytic profile of leukocytes in perivascular cuffs, we analyzed LDHA expression in the brains of 4 MS subjects of different ages (39-82 years; Supplemental Figure 11, A-D). Notably, we found that approximately $70 \%$ of the leukocytes within the active cuffs of all the brains analyzed were $\mathrm{LDHA}^{+}$(Supplemental Figure 11E), suggesting that infiltration into active lesions has high metabolic demands, irrespec- 
A

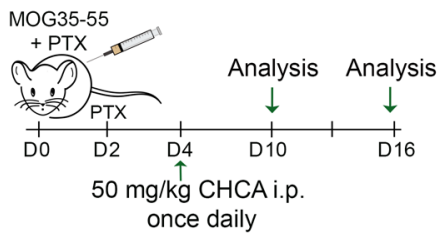

D

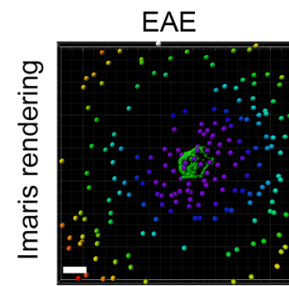

G

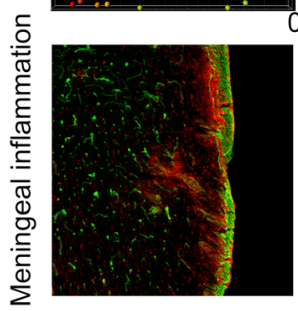

J

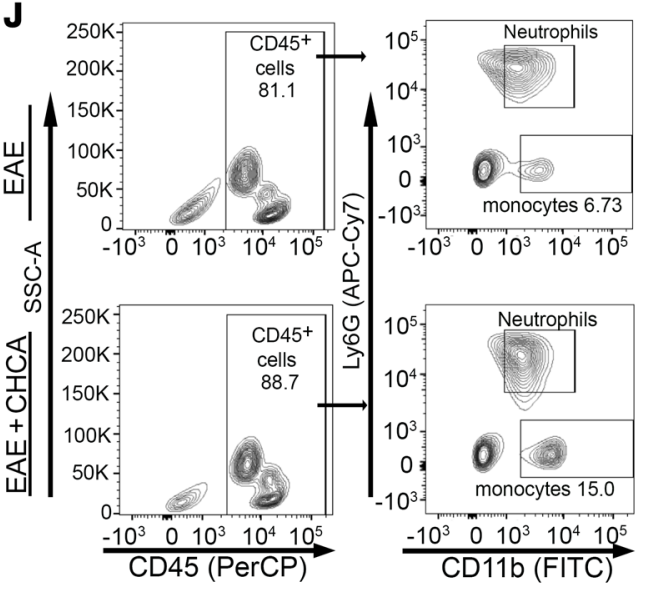

$\mathrm{EAE}+\mathrm{CHCA}$

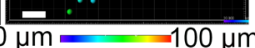

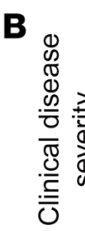
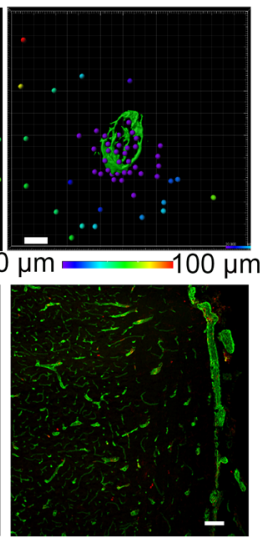

CD11b (FITC)

E

H

K

L
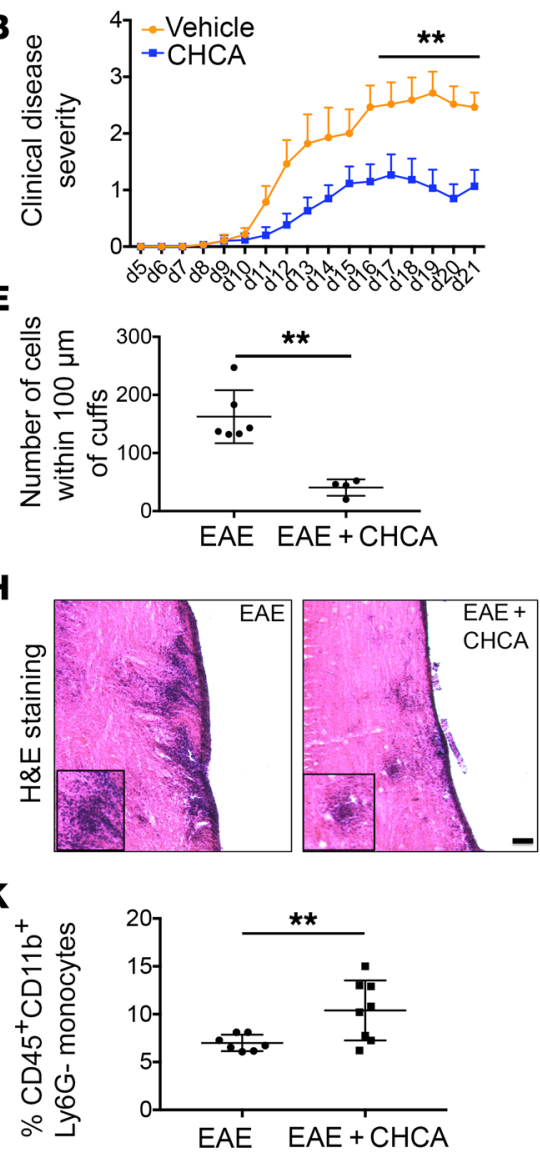

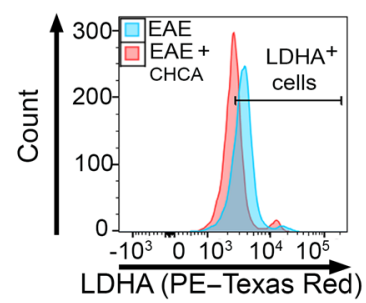

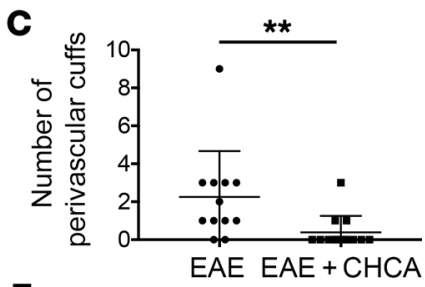

$\mathbf{F}$

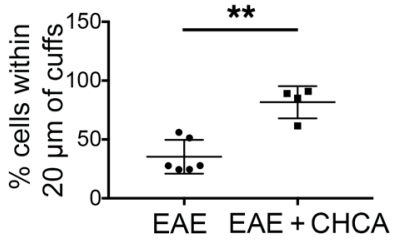

I

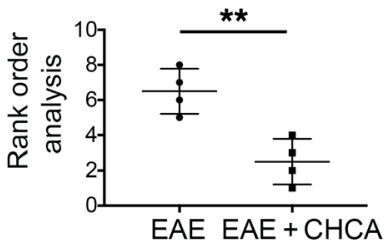

M

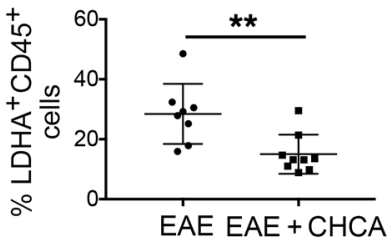

$\mathbf{N}$

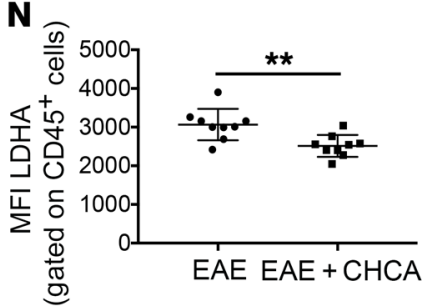

Figure 8. CHCA reduces CNS infiltration of leukocytes and decreases the disease severity of EAE. (A) Schematic shows the timeline of CHCA injections in EAE mice. (B) Clinical disease scores for CHCA- or vehicle-treated EAE mice. Results are representative of 3 independent EAE experiments (from D16 and D21 EAE mice). Plot represents 2 similarly designed independent EAE experiments combined (D21 data sets). $n=17$ mice per group, and the means of each group were compared using a 2-way ANOVA with Dunnett's post hoc multiple comparisons test. Data represent the mean \pm SEM. (C) Total number of perivascular cuffs counted across 2 sections of at least 6 EAE D16 spinal cords. (D) Images depict the distance transformation (Imaris) of representative perivascular cuffs and infiltrating $\mathrm{CD} 45^{+}$cells (spheres) in vehicle- or CHCA-treated D16 spinal cords. Cuffs were across 2 spinal cord sections of at least 3 mice analyzed. Scale bars: 20 $\mu \mathrm{m}$. (E and F) Graphs show the (E) number of cells within $100 \mu \mathrm{m}$ or $(\mathbf{F})$ percentage of cells within $20 \mu \mathrm{m}$ of the cuff. (G-I) Meningeal inflammation studied by (C) immunofluorescence using laminin (green) and CD45 (red) markers or (H) H\&E staining. Insets depict a magnified inflamed region within the EAE spinal cords. Scale bars: $100 \mu \mathrm{m}(\mathbf{G})$ and $75 \mu \mathrm{m}(\mathbf{H})$; original magnification, $\times 20$ (enlarged insets). (I) Micrographs of H\&E staining were blinded and rank ordered (Mann-Whitney rank order analysis) from 2 spinal cord sections per mouse ( $n=4$ mice per group). (J) Flow cytometric plots of CD11b+Ly6C- monocytes (gated on CD45 ${ }^{+}$cells) in blood. (K) Significant increase in the proportion of monocytes in blood of CHCA-treated EAE mice; $n=7-8$ mice per group. (L) Graph shows the overlay of LDHA in CD45+ leukocytes in the EAE and EAE plus CHCA treatment groups ( $n=8-9$ mice per group). (M) Percentage of LDHA+CD45+ cells and (N) MFI of LDHA in $\mathrm{CD}_{4} 5^{+}$cells in blood. Graphs show the mean \pm SD. Data were compared with a 2-tailed Student's $t$ test unless otherwise indicated. ${ }^{* *} P<0.01$.

tive of the duration of the disease. As described in the literature (36), we also found MCT- 4 to be present in $\mathrm{GFAP}^{+}$reactive astrocytes in the active MS lesions (Supplemental Figure 10C).

\section{Discussion}

The perivascular cuffs of postcapillary venules are one of the major routes for leukocytes such as $\mathrm{T}$ cells and macrophages to enter the CNS during EAE and MS pathogenesis. Although myelin-reactive $\mathrm{T}$ cells are considered to be the major contributor to MS pathology and depend on aerobic glycolysis for their differentiation (37), macrophages constitute the predominant population of leukocytes at perivascular cuffs with the potential to aid $\mathrm{T}$ cell migration and activation $(24,38)$. Recent evidence suggests that proinflammatory macrophages utilize glycolysis and not oxidative phosphorylation as a major source of energy (9). Indeed, we have now determined that macrophages within inflamed perivas- 


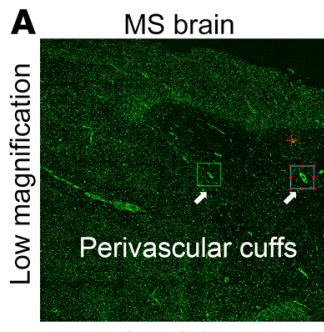

Laminin

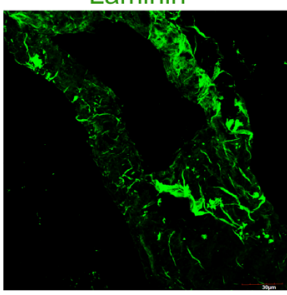

CD45

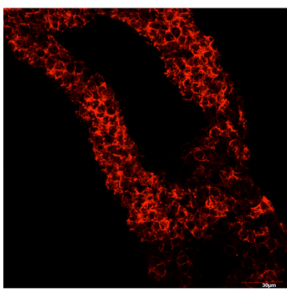

Merged

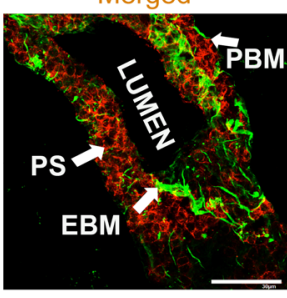

B

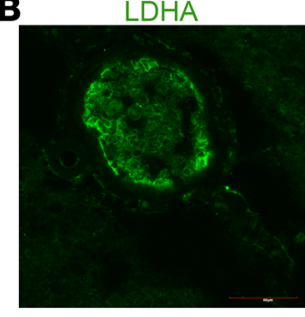

CD68

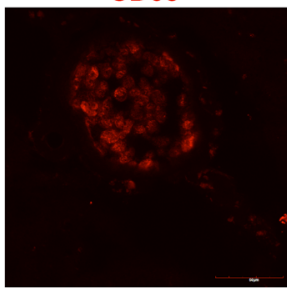

Merged

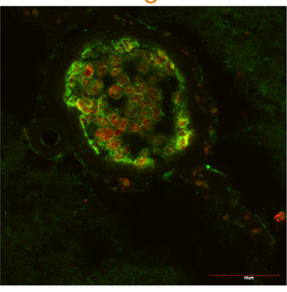

Merged/Hoechst

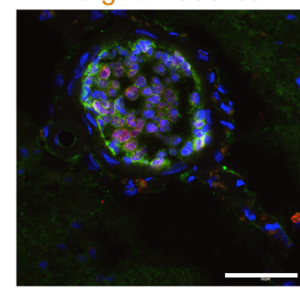

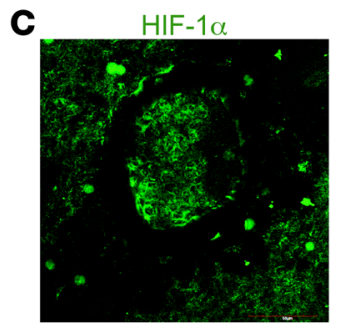

CD68

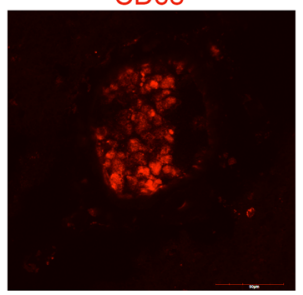

Merged

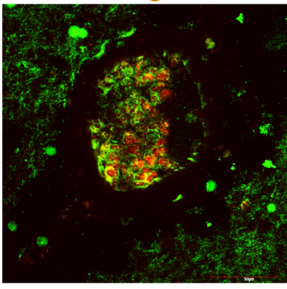

Merged/Hoechst

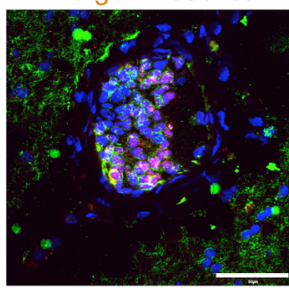

D

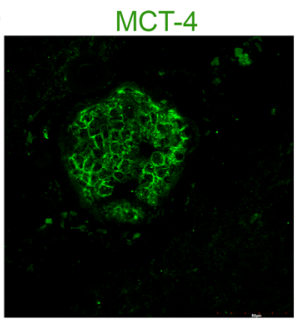

CD68

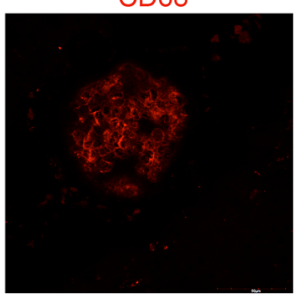

Merged

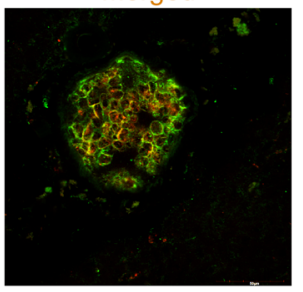

Merged/Hoechst

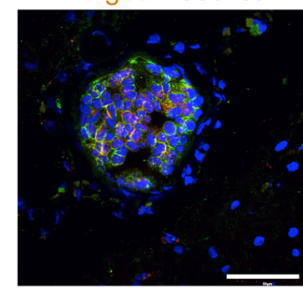

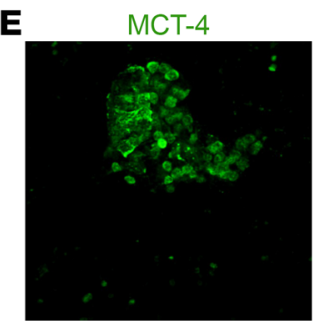

EMMPRIN

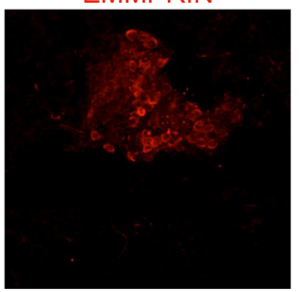

Merged

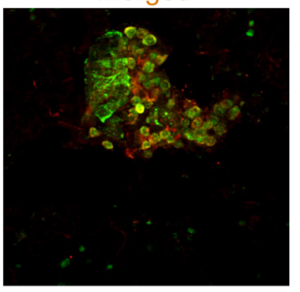

Merged/Hoechst

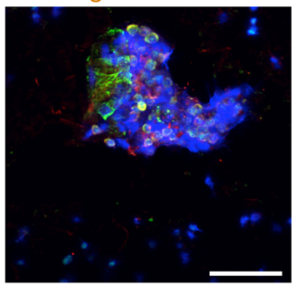

Figure 9. Infiltrating macrophages in active MS cuffs express LDHA and other glycolytic markers including HIF-1 $\alpha$ and MCT-4 and EMMPRIN. (A) Lowmagnification micrographs of an active lesion of an MS brain; 2 different active lesions per MS brain were analyzed. Arrows in the merged image point to perivascular cuffs, one of which is represented with laminin (green) and CD45 (red). The merged image identifies different regions: EBM, endothelial basement membrane; PBM, parenchymal basement membrane; PS, perivascular space within a cuff (arrows). (B-D) CD68 macrophages (red) expressing (B) LDHA (green), (C) HIF-1 $\alpha$ (green), and (D) MCT-4 (green) within an active MS lesion. (E) EMMPRIN is colocalized with MCT-4 in one such active lesion. Scale bars: $50 \mu$ m.

cular cuffs express high levels of LDHA, indicating the generation of lactate within these cells. Our studies using FX11 confirmed that LDHA activity is indeed important for the proinflammatory functions of macrophages. The Ldha gene bears hypoxia regulatory elements (HREs) in its promoter sequence $(39,40)$, suggesting that it is probably regulated by HIF- $1 \alpha$. This transcription factor in turn can also influence the expression of MCT-4 $(41,42)$, potentially by its effects on EMMPRIN (43), and it is intricately linked with metabolic reprogramming during inflammation (44). On this point, we found that the infiltrating leukocytes expressed HIF-1 $\alpha$ along with MCT-4 and its ancillary protein, EMMPRIN. The stabilization of HIF-1 $\alpha$ in autoimmune diseases has been attributed to mild hypoxia that is generated as a result of increased oxygen demand in the tissues locally (45). However, it is also noteworthy that a glycolytic shift occurs in the tissues even in normoxic conditions, an example of which is LPS-induced HIF-1 $\alpha$ stabilization (inflammatory hypoxia) without apparent hypoxia. Therefore, the relevance of hypoxia-induced HIF-1 $\alpha$ stabilization to the underlying glycolytic shift in macrophages requires further investigation.
It is noteworthy that the leukocytes that have migrated into the parenchyma seemed to be devoid of either LDHA or MCT-4 expression, suggesting that their metabolic needs may differ from those of leukocytes that accumulate in areas of cuffs. We also found a subset of leukocytes in the perivascular cuffs that expressed MCT-1 (Supplemental Figure 5B), which is another lactate exporter on macrophages. However, MCT-4 is suggested to be crucial for the secretion of large amounts of lactate from highly glycolytic cells $(32,33)$, particularly due to its lower affinity for lactate over MCT-1 (46). Furthermore, it is also understood that the switch to glycolysis by EMMPRIN involves MCT-4 and not MCT-1 (47). The expression of MCT- 4 in both the EAE and MS brains affirms the importance of this transporter in regulating lactate levels in macrophages. Recently, neuroimaging using hyperpolarized ${ }^{13} \mathrm{C}$ magnetic resonance spectroscopy in the cuprizone mouse model of MS demonstrated that areas rich in infiltrating macrophages had higher rates of lactate production (48), thus corroborating our findings of the importance of lactate metabolism for macrophage transmigration into the CNS. Knocking down MCT- 4 has been described 
in the literature to reduce glycolysis via reduction of hexokinase 2 and 6-phosphofructo-2-kinase/fructose-2, 6-biphosphatase 3 in activated macrophages (34). In addition, we found that MCT-4 knockdown resulted in a reduction of LDHA expression, and thus lactate generation, in LPS-stimulated BMDMs. This impaired the production of TNF- $\alpha$ and migration of BMDMs in vitro, the key proinflammatory features of macrophages.

Our current observations implicate EMMPRIN as a chaperone to MCT-4 within macrophages that enter the perivascular cuffs of EAE and MS brains. Moreover, the EMMPRIN/MCT-4 axis plays a crucial role in the maintenance of LDHA-dependent lactate production in inflammatory macrophages. In support of these observations, we found that genetic knockdown of MCT-4 as well as functional inhibition of MCT- 4 with CHCA reduced the migratory function of macrophages, and this was underscored by decreased EMMPRIN-MCT-4 interaction. The molecular forms of EMMPRIN range from $27 \mathrm{kDa}$ (unglycolysated protein) to higher than $80 \mathrm{kDa}$, depending on the complexity of glycosylation (49, 50). Notably, we observed changes in several glycosylated forms of EMMPRIN (up to $80 \mathrm{kDa}$ ) in EAE spinal cords; enrichment of protein fractions from macrophages upon co-IP yielded other relevant glycosylated EMMPRIN forms. Our findings suggest that while a glycosylated form of EMMPRIN of approximately $50 \mathrm{kDa}$ interacted with MCT-4, a higher glycosylated form of EMMPRIN (>100 kDa; likely a multimeric form of glycosylated EMMPRIN) was particularly sensitive to the inflammatory status of macrophages. The observations that knockdown of EMMPRIN exerted effects similar to those of functional inhibition of MCT-4 on decreased glycolytic functions and impaired TNF- $\alpha$ production as well as BMDM migration in activated macrophages suggest the importance of EMMPRIN in maintaining key biological functions, much like its effects in other cell types. Indeed, EMMPRIN-null mice have reduced survival rates (51) and impaired sensory and memory functions $(52,53)$, suggesting the crucial role of EMMPRIN in the maintenance of homeostasis.

Diet plays an important role in the outcome of inflammatory disorders $(54,55)$. It is noteworthy that cinnamic acid is a major ingredient of cinnamon and turmeric, which are major dietary components in several parts of the world. Both cinnamon and turmeric are widely used as antiinflammatory agents in the form of flavoring agents and spices $(56,57)$. Of relevance to MS, ground cinnamon can alleviate disease activity in EAE by bolstering the Treg population, leading to decreased EAE severity (55). However, cinnamon is a complex mixture of cinnamaldehyde and cinnamic acid, among other metabolites. Therefore, the key metabolites responsible for the observed effects on Tregs and other leukocyte populations have yet to be identified. We found that CHCA (an analog of cinnamic acid) decreased disease severity in EAE mice by interfering with the EMMPRIN/MCT-4 axis that shuttles lactate out of macrophages. In addition, we found that CHCA also reduced the infiltration of $\mathrm{CD}^{+} \mathrm{T}$ cells across the perivascular cuffs during peak EAE, possibly by dampening the secretion of proinflammatory cytokines by macrophages as noted in LDHA-inhibitory conditions. Indeed, when we analyzed the expression of LDHA in peripheral T cells, we did not detect elevation of LDHA expression in peripheral T cells, opposite to what we observed in monocytoid cells. This is a surprising finding, as pathogenic T cells (Th1 and Th17) are known to undergo a glycolytic shift in MS $(58,59)$. One reason could be that we studied T cells in the periphery, where the glycolytic shift may occur at a different time point in the disease course in a T cell subset-specific manner. Also, the pathogenic T cells entering the CNS compartments during EAE exhibit shift to the glycolytic profile as compared with the ones in the blood $(58,59)$.

Further analysis of direct effects of CHCA on T cell polarization generated complex data sets. On the one hand, CHCA decreased the IFN- $\gamma$-producing Th1 cells in anti-CD3/anti-CD28 polyclonally activated splenocytes (Supplemental Figure 9C), without altering their proliferation (Supplemental Figure 9B); on the other hand, CHCA enhanced IFN- $\gamma^{+}$T-bet $^{+}$Th1 cells during the Th1-polarizing experiments (Supplemental Figure 9, E and F). Interestingly, no effects of CHCA on Th17 polarization were noted (Supplemental Figure 9, D, G, and H). Importantly, the functions of CHCA are grossly antiinflammatory, as treatment with CHCA resulted in a reduction of EAE disease severity. These results add another dimension to the roles that dietary components play in inflammatory disorders, and they highlight a mechanism by which cinnamon derivatives may influence MS outcomes.

According to existing literature and our observations, the production and export of lactate serves 2 important functions: first, it provides a feed-forward signal for glycolysis to continue uninhibited, leading to the production of proinflammatory cytokines; and second, lactate can lead to the generation of a Th17 subset of CD $4^{+}$ T cells, which are known to exacerbate MS and EAE (60). Furthermore, lactate can boost LPS-stimulated TLR4 signaling in macrophages and the subsequent production of inflammatory cytokines at least in human monocyte-derived macrophages (13). However, lactate has also been reported to dampen LPS-induced inflammation in macrophages (14), which is possibly exerted by GPR81, a receptor for lactate on macrophages (61). The immunosuppressive functions of lactate are also described extensively in the tumor literature, where it is shown to upregulate HIF- $1 \alpha$-dependent increases in arginase- 1 as well as ERK/STAT3 signaling pathways $(62,63)$. Therefore, the autocrine/paracrine immunomodulatory effects of lactate secreted by macrophages in inflammatory conditions such as MS require further investigation.

Given that blocking MCT-4 functions on macrophages alleviated their activation and modulated the severity of EAE disease, interfering with the glycolytic functions is an attractive therapy to treat inflammatory conditions such as MS. A recent study by Kornberg et al. (64) showed that the MS drug dimethyl fumarate and its metabolite monomethyl fumarate act by inactivating the glycolytic enzyme glyceraldehyde 3-phosphate dehydrogenase in peritoneal macrophages and $\mathrm{CD}^{+} \mathrm{T}$ cells in both mice and humans. Similarly, glatiramer acetate (Copaxone) modulates immune infiltration in part by decreasing glycolysis within the T cells of patients with relapsing-remitting MS (RRMS) (65). Thus, targeting glycolysis in immune subsets may represent a viable therapy to treat MS. However, it is important to keep in mind the role lactate may serve centrally during an ongoing pathology, as the lactate produced by CNS cells such as astrocytes and oligodendrocytes can serve as an additional energy source for neurons and oligodendrocytes during stress. Therefore, further validation and studies examining the feasibility of long-term lactate/glycolysis targeting are necessary to unravel the true benefits of this approach. 
In summary, our study highlights the importance of EMMPRIN and MCT-4 in governing macrophage glycolysis within inflammatory perivascular cuffs in EAE and MS and how dietary factors, especially cinnamon derivatives, may affect these molecular interactions to reduce the leukocyte trafficking and neuroinflammation relevant to MS.

\section{Methods}

EAE induction. Eight- to ten-week-old C57BL/6 female mice (Charles River Laboratories) were immunized s.c. with $50 \mu \mathrm{g} / 100 \mu \mathrm{L}$ MOG 35-55 peptide in CFA supplemented with $4 \mathrm{mg} / \mathrm{mL}$ heat-inactivated Mycobacterium tuberculosis H37Ra (Thermo Fisher Scientific); $50 \mu \mathrm{L}$ emulsion was deposited on either side of the tail base. Pertussis toxin (PTX) (300 ng/200 $\mu \mathrm{L} ; 180$, List Biological Laboratories) was injected i.p. on days 0 and 2 after MOG immunization. CHCA (C2020, MilliporeSigma) was dissolved in PBS, and the $\mathrm{pH}$ was adjusted to approximately 7.4 with $1 \mathrm{~N} \mathrm{NaOH}$ and administered i.p. (in $100-\mu \mathrm{L}$ volumes) starting on D4 after MOG immunization. CHCA was given once daily at a dose of 25 or $50 \mathrm{mg} / \mathrm{kg}$ until the experiment was terminated. A similar volume of PBS was administered i.p. as control injections. Three EAE experiments were conducted independently. Daily monitoring of EAE mice was performed, and the mice were scored on a scale of 0 to 5 .

Tissue harvesting and flow cytometry. Peripheral monocytes from EAE and healthy mice that were anesthetized with a lethal dose of ketamine and xylazine were harvested from whole blood isolated by cardiac puncture. Approximately $0.5 \mathrm{~mL}$ heparinized whole blood was collected from each mouse, and RBC lysis was carried out using RBC lysis solution (555899, BD Biosciences) before proceeding with flow cytometry.

For the myelin removal protocol, myelin from spinal cord homogenates was isolated using a Percoll gradient as described earlier (1). Flow cytometry was performed using fluorescence-conjugated antibodies against CD45-PerCP (553310, BD Biosciences, 1:50); LDHA monoclonal antibody (AF14A11; MA5-17247, Thermo Fisher Scientific, 1:50); CD11b-FITC (557235, BD Biosciences, 1:50); and Ly6G-APC-Cy7 (560600, BD Biosciences, 1:50). LDHA antibody was conjugated with DyLight 594 using an antibody conjugation kit (ab201801, Abcam). For staining, the cells were suspended in FACS buffer and blocked with the Fc-blocker CD16/CD32 (553141, BD Biosciences, 1:100) for 20 minutes at $4^{\circ} \mathrm{C}$. Cells were washed in FACS buffer and incubated in diluted antibodies for 30 minutes at $4^{\circ} \mathrm{C}$ in the dark. Cells were washed in FACS buffer and fixed in $1 \%$ formalin before being suspended in FACS buffer and analyzed using a BD LSR II FACS system.

Splenocyte proliferation and $T$ cell polarization. Splenocytes isolated from the spleens of 6- to 8-week-old female C57BL/6 mice were seeded in RPMI 1640 media supplemented with 10\% FBS (Thermo Fisher Scientific) at $10^{5}$ cells/well in 96-well round-bottomed plates that were coated with $1 \mu \mathrm{g} / \mathrm{mL}$ anti-mouse CD3 antibody (553058, BD Biosciences) for 2 hours at $37^{\circ} \mathrm{C}$. Anti-mouse CD28 antibody (553295, $\mathrm{BD}$ Biosciences) was then added to the wells at a final concentration of $1 \mu \mathrm{g} / \mathrm{mL}$. The cells were then treated with 200 and $400 \mu \mathrm{M} \mathrm{CHCA}$ for 24 hours, after which proliferation was measured on a scintillation beta counter by adding ${ }^{3} \mathrm{H}$-thymidine (PerkinElmer) at a final concentration of $1 \mu \mathrm{Ci}$ per well.

For Th1 and Th17 polarization experiments, $\mathrm{CD} 4^{+} \mathrm{T}$ cells were purified from the spleens of 6- to 8-week-old female C57BL/6 mice using the EasySep Mouse CD4 ${ }^{+}$T Cell Kit (19852, STEMCELL Technologies) and activated with Dynabeads Mouse T-Activator CD3/
CD28 (11456D, Thermo Fisher Scientific). Th0 cocktail $(500 \mu \mathrm{L})$ containing IL-2 (5 ng/mL, 212-12, Peprotech), anti-IFN- $\gamma$ antibody $(10 \mu \mathrm{g} / \mathrm{mL}, 505802$, BioLegend), and anti-IL-4 antibody $(10 \mu \mathrm{g} / \mathrm{mL}$, 504102, BioLegend); or Th1 cocktail containing IL-2 (5 ng/mL, 21212, Peprotech), IL-12p70 (10 ng/mL, 210-12, Peprotech), and antiIL-4 (10 $\mu \mathrm{g} / \mathrm{mL}, 504102$, BioLegend); or Th17 cocktail containing IL-6 (50 ng/mL, 216-16, Peprotech), TGF- $\beta$ (1 ng/mL, 7666-MB-005, R\&D Systems), IL-23 (5 ng/mL, 589002, BioLegend), anti-IFN- $\gamma$ (10 $\mu \mathrm{g} / \mathrm{mL}, 505802$, BioLegend), and anti-IL-4 antibody $(10 \mu \mathrm{g} / \mathrm{mL}$, 504102, BioLegend) was added to the wells in the presence of DMSO (control) or 200 or $400 \mu \mathrm{M}$ CHCA. Following 4 days of incubation, 2 $\mu \mathrm{L}$ Leukocyte Activation Cocktail with GolgiPlug (550583, BD Biosciences) was added to each well for 5 hours, and FACS staining was performed using the following antibodies (all from BD bioscience unless otherwise indicated): anti-CD3 (FITC, 561827); anti-CD4 (APC-Cy7, 552051); anti-T-bet (BV421, 563318); anti-IFN- $\gamma$ (PE, 554412); antiROR $\gamma \mathrm{t}$ (BV421, 562894); and anti-IL-17A (APC, 17-7177-81, eBioscience). Viable cells were gated using Fixable Viability Stain 620 (56499, BD Biosciences). FACS data was acquired on the BD LSRII instrument (BD Biosciences), and data was analyzed using FlowJo software, version 10 (Treestar).

BMDM isolation and treatment. BM from 6- to 8-week-old C57Bl/6 mice was harvested by flushing the femur and tibia, and the cells were seeded at a density of $10^{7}$ cells per bacterial Petri dish and grown in DMEM in the presence of $10 \%$ L929 conditioned supernatant and 10\% FBS along with $2 \%$ penicillin-streptomycin for 1 week as described previously (66). The BMDMs were treated with $100 \mathrm{ng} / \mathrm{mL}$ LPS in the presence or absence of 200 or $400 \mu \mathrm{M}$ CHCA and $20 \mu \mathrm{M}$ FX11 (427218, MilliporeSigma). Control cells were treated with a similar volume of DMSO.

For knockdown studies, BMDMs were transfected with either 50 nM scrambled RNA (sc-37007); LDHA siRNA (ID 156092, Thermo Fisher Scientific); MCT-4 siRNA (ID 174958, Thermo Fisher Scientific); or EMMPRIN siRNA (sc-35299, Santa Cruz Biotechnology) using Lipofectamine 2000 (Thermo Fisher Scientific) according to the manufacturer's protocol. Transfection was carried out for at least 36 hours before membrane isolation or measurement of ECAR activity and for at least 48 to 72 hours for measurement of lactate.

Protein isolation and co-IP. The treated BMDMs were lysed for total protein using RIPA buffer (89900, Pierce, Thermo Fisher Scientific) containing protease and phosphatase inhibitors (Cell Signaling Technology). The lysates were centrifuged at $13,000 \mathrm{~g}$ for 10 minutes, and supernatants were collected. Membrane protein was isolated using the membrane isolation kit according to the manufacturer's protocol (89842, Thermo Fisher Scientific), and proteins were estimated using the bicinchoninic acid (BCA) method (MilliporeSigma).

For co-IP experiments, $500 \mu \mathrm{g}$ total lysate was incubated with 1 $\mu \mathrm{g}$ anti-MCT-4 antibody (sc-50329; Santa Cruz Biotechnology) or isotype IgG control (ab27472; Abcam) overnight at $4^{\circ} \mathrm{C}$ to form the immune complex. The IP assay was performed using the Magnetic IP Kit (88804, Thermo Fisher Scientific) according to the manufacturer's protocol. Briefly, the antigen-antibody mixture was incubated with the magnetic beads prewashed in IP lysis and wash buffer for 1.5 hours. Then, the beads were collected using a magnetic stand, and the unbound sample was collected for analysis. The beads were then washed and eluted in $50 \mu \mathrm{L}$ low-pH elution buffer. The eluate along with the unbound fraction was then subjected to Western blot analysis. 
Western blot analysis. The BMDM lysates (30 $\mu$ g per lane) and both the unbound fractions and the eluate from co-IP were loaded onto a $10 \%$ polyacrylamide gel and transferred onto PVDF membranes. The membranes were then blocked with either 5\% milk or Startblock T20 buffer (Thermo Fisher Scientific; for co-IP blots) for 1 hour and then incubated with the following antibodies overnight at $4^{\circ} \mathrm{C}$ : rabbit anti-EMMPRIN (42-58 kDa, ab108317, Abcam, 1:1000); rat anti-EMMPRIN (50 kDa, OX114, MCA2283, 1:500, Bio-Rad Laboratories); rabbit anti-MCT-4 (SC-50329, Santa Cruz Biotechnology, 1:500); rabbit anti-HIF-1 $\alpha$ (NB100-134, Novus Biologicals, 1:1000); anti-rabbit LDHA (NBP1-48336, Novus Biologicals, 1:1000); anti-GFAP (Z0334, Dako, 1:400); anti-Na-K $\mathrm{K}^{+}$ATPase (ab76020, Abcam, 1: 10,000); and $\beta$-actin (ab20272, Abcam, 1:5000) in 1\% skim milk. The following day, membranes were washed with 0.1\% $1 \times$ TBS Tween-20 (TBST) and incubated with the corresponding HRP-linked secondary antibody (Jackson ImmunoResearch Laboratories, 1:5000) or Clean-Blot Reagent (1:80; Thermo Fisher Scientific; for co-IP blots) for 1.5 hours in TBST for chemiluminescence imaging (Bio-Rad Laboratories).

MS specimens. Postmortem brain tissues from individuals with chronic MS were obtained from the UK Multiple Sclerosis Tissue Bank at Imperial College, London (www.ukmstissuebank.imperial.ac.uk; provided by Richard Reynolds and Djordje Gveric). For this study, we assessed donor brain tissues from 4 patients with MS (1 male and 3 female donors; age range: 39-82 years): 1 who had RRMS, 2 who had secondary progressive MS (SPMS), and 1 whose MS was not specified. For our purposes, we only studied active cuffs with infiltrating leukocytes.

Fluorescence microscopy and 3 D reconstruction. Mice were perfused with ice-cold PBS, and the spinal cords and cerebella were harvested and fixed in OCT medium. Tissues were then sectioned at 20- to $30-\mu \mathrm{m}$ thickness, fixed in methanol at $-20^{\circ} \mathrm{C}$, and incubated with rabbit anti-pan laminin (a gift from L. Sorokin, University of Münster, Münster, Germany; 1:1000); rat anti-mouse CD45 (clone 30F11, 550539, BD Pharmingen, 1:100); rabbit anti-LDHA (NBP1-48336, Novus Biologicals, 1:100); rabbit anti-MCT-4 (sc-50329, Santa Cruz Biotechnology, 1:50); rat anti-EMMPRIN (clone OX114, MCA2283, Bio-Rad Laboratories, 1:30); rabbit anti-HIF-1 $\alpha$ (NB100-134, Novus Biologicals, 1:100); mouse anti-human CD68 (V1/824, 556059, BD Pharmingen, 1:100); anti-CD3 (ab11089, Abcam, 1:100); anti-F4/80 (MCA497EL; BD Biosciences); rabbit anti-GFAP (G3893, MilliporeSigma 1:400); or mouse anti-MCT-1 (ab90582, Abcam, 1:100) antibodies overnight at $4^{\circ} \mathrm{C}$. After additional washes, sections were incubated with Alexa Fluor 488- and Alexa Fluor 546-conjugated secondary antibodies (Jackson ImmunoResearch Laboratories, 1:500). The nuclei were stained with nuclear yellow (Hoechst), and MS brains were incubated for an additional 30 seconds with Sudan black dye to quench signals from endogenous lipofuscin granules. Images were captured on a Fluoview FV10i confocal microscope (Olympus).

3D reconstruction of the $Z$-stacks from static confocal images was performed using Imaris 3D reconstruction software (Bitplane), in which surfaces were created for each channel and then a merged surface reconstructed. For colocalization analysis, a colocalization channel was created by setting thresholds on the merged images, and the percentage of colocalization between different channels was reported.

Real-time PCR. BMDMs were homogenized in $1 \mathrm{~mL}$ TRIzol Reagent (Thermo Fisher Scientific), and RNA was isolated using the RNeasy Mini Kit (74104, QIAGEN) following the manufacturer's instructions. Real-time PCR (RT-PCR) was performed on the
QuantStudio 6 Flex (Applied Biosystems) using Fast SYBR Green (Thermo Fisher Scientific), transcripts were analyzed by the $\Delta \Delta \mathrm{Ct}$ method, and the data were normalized to Gapdh or Actb. Primers for Gapdh (QIAGEN ref. QT01658692; NCBI RefSeq: NM_008084); Ldha (QIAGEN ref. QT02325414; NCBI RefSeq: NM_010699); MCT-4 (QIAGEN ref. QT00116039; NCBI RefSeq: NM_030696); and Actb (QIAGEN ref. QT00095242; NCBI RefSeq: NM_007393) were obtained from QIAGEN. The primer sequences for EMMPRIN (basigin isoform 2; NCBI RefSeq: NM_001077184.1) were as follows: forward, 5'-GCGGCGGGCACC ATCCAA AC-3'; reverse, 5'-ATGTACTTCGTATGCAGGTCG G-3'.

Live/dead assay and ImageXpress. BMDMs were seeded in a 96-well plate at a density of 50,000 cells per well in a black-bottomed plate (BD Biosciences). After BMDMs were treated for 24 hours with $100 \mathrm{ng} / \mathrm{mL}$ LPS, either in the presence or absence of different concentrations of CHCA and FX11, $20 \mu \mathrm{L}$ of a mixture containing nuclear yellow dye (NucBlue, Thermo Fisher Scientific), $5 \mu \mathrm{M}$ calcein AM (Invitrogen, Thermo Fisher Scientific), and $5 \mu \mathrm{g} /$ $\mathrm{mL}$ propidium iodide (PI) (MilliporeSigma) was added directly to the media for 20 minutes. Images of calcein $\mathrm{AM}^{+}$(green; live cells) and $\mathrm{PI}^{+}$cells (red; dead cells) were captured using the live image module of ImageXpress (Molecular Devices), and the percentage of live and dead cells was calculated using MetaXpress acquisition and analysis software (Molecular Devices). MCT-4-stained BMDMs were also captured using ImageXpress, and the fluorescence per cell was quantified using Fiji software (ImageJ, NIH).

Lactate measurement. BMDMs were treated or siRNA transfected in 0.5\% FBS DMEM (without L929 supernatant supplementation and without sodium pyruvate), and 48 hours or 72 hours later, the BMDMs were treated with LPS for another 18 to 24 hours, followed by collection of the supernatant. Lactate from cell culture supernatant was measured using the fluorescence-based lactate measurement kit according to the manufacturer's protocol (ab65330, Abcam). Similarly, lactate from spinal cord lysates was measured and normalized to the protein concentration. Briefly, cell culture supernatants (1:200 primary dilution) and spinal cord lysates (1:500 primary dilution) and the standards were incubated in the presence of the lactate probe and enzyme mix at room temperature for 30 minutes and measured at $535 / 587 \mathrm{~nm}$ excitation/emission. Lactate levels were calculated from the standard curve and are represented in millimolars.

Luminex assay and ELISA. TNF- $\alpha$ was measured from the supernatants of BMDMs conditioned with different treatments using an ELISA kit (BMS607-3, Thermo Fisher Scientific) according to the manufacturer's protocol. For the Luminex assay, $50 \mu \mathrm{L}$ cell culture supernatant was analyzed using the 31-Plex Mouse Cytochemokine Array (MD31, Eve Technologies). For inhibitor experiments, BMDMs were stimulated with $100 \mathrm{ng} / \mathrm{ml}$ LPS in the presence of inhibitors for 24 hours. However, for measurement of TNF- $\alpha$ in knockdown conditions, BMDMs were stimulated for 6 hours with $50 \mathrm{ng} / \mathrm{mL}$ LPS.

ECAR measurement. ECAR was determined using the Seahorse XFe24 Flux Analyzer (Agilent Technologies). Briefly, the cells were seeded at a density of $2.5 \times 10^{5}$ per well the day before the assay and treated with LPS in the presence or absence of CHCA or FX11, or subjected to transfection. The media were changed to the XF assay media (Agilent Technologies) either after 12 hours (for CHCA) or 18 hours (for FX11 and siRNA transfection) of LPS treatment and run to assess ECAR with the addition of $25 \mathrm{mM}$ glucose, $1 \mu \mathrm{M}$ oli- 
gomycin (Enzo Life Sciences), and $100 \mu \mathrm{M}$ 2-deoxyglucose (2DG) (MilliporeSigma), in ports A, B and C, respectively. Seahorse Wave software was used to analyze the data, which were normalized to the amount of protein in micrograms. The parameter equations used for the calculation of glycolysis and glycolytic capacity were as follows: [(measurement before oligomycin injection) - (measurement after 2DG injection)] and [(measurement after oligomycin) - (measurement after 2DG injection)], respectively.

Statistics. All results are expressed as the mean \pm SD unless otherwise stated. Statistical analysis was performed with GraphPad Prism 7.0 (GraphPad Software). Comparisons of 2 groups were done using a 2-tailed Student's $t$ test, and multiple means were compared using either a 1-way ANOVA with Tukey's post hoc test or a 2-way ANOVA with Dunnett's post hoc multiple comparisons test (EAE time course experiments). $P$ values of less than 0.05 were considered significant.

Study approval. All experiments were conducted in accordance with guidelines of the Canadian Council on Animal Care and with ethics approval from the Animal Care Committee at the University of Calgary. All MS tissues were obtained and used with approval from the institutional ethics committee of the University of Calgary.

\section{Author contributions}

DKK and VWY conceived the study and drafted the manuscript. DKK performed experiments and analyzed data. AB, KSR, RM, and YA carried out experiments and helped with the editing of the manuscript. JMR provided resources for the Seahorse experiments and edited the manuscript. All the authors read and approved the final version of this manuscript.

\section{Acknowledgments}

This study was supported by grants from the Canadian Institute of Health Research and Alberta Innovates - Health Solutions (AIHS) (CRIO Team program). DKK acknowledges postdoctoral fellowship funding from AIHS and the Multiple Sclerosis Society of Canada. DKK also received a fellowship from the University of Calgary's Eyes High program. KSR is a Canada Varnier Scholar, and RM was supported by a fellowship from the University of Calgary's Eyes High program. VWY holds a Tier 1 Canada Research Chair.

Address correspondence to: V. Wee Yong, University of Calgary, 3330 Hospital Drive, Calgary, Alberta T2N 4N1, Canada. Phone: 403.220.3544; Email: vyong@ucalgary.ca.
1. Agrawal SM, Silva C, Tourtellotte WW, Yong VW. EMMPRIN: a novel regulator of leukocyte transmigration into the CNS in multiple sclerosis and experimental autoimmune encephalomyelitis. J Neurosci. 2011;31(2):669-677.

2. Agrawal SM, et al. Extracellular matrix metalloproteinase inducer shows active perivascular cuffs in multiple sclerosis. Brain. 2013;136(Pt 6):1760-1777.

3. Song J, et al. Focal MMP-2 and MMP-9 activity at the blood-brain barrier promotes chemokine-induced leukocyte migration. Cell Rep. 2015;10(7):1040-1054.

4. Rua R, McGavern DB. Advances in Meningeal Immunity. Trends Mol Med. 2018;24(6):542-559.

5. Engelhardt B, Vajkoczy P, Weller RO. The movers and shapers in immune privilege of the CNS. Nat Immunol. 2017;18(2):123-131.

6. van Zwam M, et al. Myelin ingestion alters macrophage antigen-presenting function in vitro and in vivo. J Leukoc Biol. 2011;90(1):123-132.

7. McMahon EJ, Bailey SL, Castenada CV, Waldner $\mathrm{H}$, Miller SD. Epitope spreading initiates in the CNS in two mouse models of multiple sclerosis. Nat Med. 2005;11(3):335-339.

8. Kelly B, O'Neill LA. Metabolic reprogramming in macrophages and dendritic cells in innate immunity. Cell Res. 2015;25(7):771-784.

9. Gaber T, Strehl C, Buttgereit F. Metabolic regulation of inflammation. Nat Rev Rheumatol. 2017;13(5):267-279.

10. Ip WKE, Hoshi N, Shouval DS, Snapper S, Medzhitov R. Anti-inflammatory effect of IL-10 mediated by metabolic reprogramming of macrophages. Science. 2017;356(6337):513-519.

11. Sotelo-Hitschfeld T, Fernández-Moncada I, Barros LF. Acute feedback control of astrocytic glycolysis by lactate. Glia. 2012;60(4):674-680

12. Le A, et al. Inhibition of lactate dehydrogenase $\mathrm{A}$ induces oxidative stress and inhibits tumor progression. Proc Natl Acad Sci USA. 2010;107(5):2037-2042.
13. Samuvel DI, Sundararaj KP, Nareika A, Lopes-Virella MF, Huang Y. Lactate boosts TLR4 signaling and NF-kappaB pathway-mediated gene transcription in macrophages via monocarboxylate transporters and MD-2 up-regulation. J Immunol. 2009;182(4):2476-2484.

14. Errea A, et al. Lactate Inhibits the Pro-Inflammatory Response and Metabolic Reprogramming in Murine Macrophages in a GPR81-Independent Manner. PLoS ONE. 2016;11(11):e0163694.

15. Brand A, et al. LDHA-Associated Lactic Acid Production Blunts Tumor Immunosurveillance by $\mathrm{T}$ and NK Cells. Cell Metab. 2016;24(5):657-671.

16. Fiume L, Manerba M, Vettraino M, Di Stefano G. Inhibition of lactate dehydrogenase activity as an approach to cancer therapy. Future Med Chem. 2014;6(4):429-445.

17. Lee Y, et al. Oligodendroglia metabolically support axons and contribute to neurodegeneration. Nature. 2012;487(7408):443-448.

18. Pierre K, Pellerin L, Debernardi R, Riederer BM, Magistretti PJ. Cell-specific localization of monocarboxylate transporters, MCT1 and MCT2, in the adult mouse brain revealed by double immunohistochemical labeling and confocal microscopy. Neuroscience. 2000;100(3):617-627.

19. Voss DM, Spina R, Carter DL, Lim KS, Jeffery CJ, Bar EE. Disruption of the monocarboxylate transporter-4-basigin interaction inhibits the hypoxic response, proliferation, and tumor progression. Sci Rep. 2017;7(1):4292.

20. Le Floch R, et al. CD147 subunit of lactate/ $\mathrm{H}+$ symporters MCT1 and hypoxia-inducible MCT4 is critical for energetics and growth of glycolytic tumors. Proc Natl Acad Sci USA. 2011;108(40):16663-16668.

21. Kirk P, Wilson MC, Heddle C, Brown MH, Barclay AN, Halestrap AP. CD147 is tightly associated with lactate transporters MCT1 and MCT4 and facilitates their cell surface expression. EMBO J. 2000;19(15):3896-3904.
22. Hahn JN, Kaushik DK, Mishra MK, Wang J, Silva C, Yong VW. Impact of Minocycline on Extracellular Matrix Metalloproteinase Inducer, a Factor Implicated in Multiple Sclerosis Immunopathogenesis. J Immunol. 2016;197(10):3850-3860.

23. Semba $H$, et al. HIF- $1 \alpha$-PDK1 axis-induced active glycolysis plays an essential role in macrophage migratory capacity. Nat Commun. 2016;7:11635.

24. Stephenson EL, et al. Chondroitin sulfate proteoglycans as novel drivers of leucocyte infiltration in multiple sclerosis. Brain. 2018;141(4):1094-1110.

25. Tannahill GM, et al. Succinate is an inflammatory signal that induces IL-1 $\beta$ through HIF-1 $\alpha$. Nature. 2013;496(7444):238-242.

26. Le Moan N, et al. Hypoxia inducible factor- $1 \alpha$ in astrocytes and/or myeloid cells is not required for the development of autoimmune demyelinating disease. eNeuro. 2015;2:e0050.

27. Grist JT, et al. Extracellular Lactate: A Novel Measure of T Cell Proliferation. JImmunol. 2018;200(3):1220-1226.

28. Shin T, Kojima T, Tanuma N, Ishihara Y, Matsumoto Y. The subarachnoid space as a site for precursor $\mathrm{T}$ cell proliferation and effector $\mathrm{T}$ cell selection in experimental autoimmune encephalomyelitis. J Neuroimmunol. 1995;56(2):171-178.

29. Ohmori K, Hong Y, Fujiwara M, Matsumoto Y. In situ demonstration of proliferating cells in the rat central nervous system during experimental autoimmune encephalomyelitis. Evidence suggesting that most infiltrating $\mathrm{T}$ cells do not proliferate in the target organ. Lab Invest. 1992;66(1):54-62.

30. Liu L, et al. Proinflammatory signal suppresses proliferation and shifts macrophage metabolism from Myc-dependent to HIF1 $\alpha$-dependent. Proc Natl Acad Sci USA. 2016;113(6):1564-1569.

31. Rellinger EJ, et al. FX11 inhibits aerobic glycolysis and growth of neuroblastoma cells. Surgery. 2017;161(3):747-752.

32. Dimmer KS, Friedrich B, Lang F, Deitmer JW, Bröer 
S. The low-affinity monocarboxylate transporter MCT4 is adapted to the export of lactate in highly glycolytic cells. Biochem J. 2000;350 Pt 1:219-227.

33. Manning Fox JE, Meredith D, Halestrap AP. Characterisation of human monocarboxylate transporter 4 substantiates its role in lactic acid efflux from skeletal muscle. JPhysiol (Lond). 2000;529(Pt 2):285-293.

34. Tan Z, et al. The monocarboxylate transporter 4 is required for glycolytic reprogramming and inflammatory response in macrophages. J Biol Chem. 2015;290(1):46-55.

35. Ge H, et al. Functional relevance of protein glycosylation to the pro-inflammatory effects of extracellular matrix metalloproteinase inducer (EMMPRIN) on monocytes/macrophages. PLoS ONE. 2015;10(2):e0117463.

36. Nijland PG, et al. Cellular distribution of glucose and monocarboxylate transporters in human brain white matter and multiple sclerosis lesions. Glia. 2014;62(7):1125-1141.

37. Peng M, Yin N, Chhangawala S, Xu K, Leslie CS, Li MO. Aerobic glycolysis promotes T helper 1 cell differentiation through an epigenetic mechanism. Science. 2016;354(6311):481-484.

38. Chitnis T, Weiner HL. CNS inflammation and neurodegeneration. J Clin Invest. 2017;127(10):3577-3587.

39. Semenza GL, et al. Hypoxia response elements in the aldolase $\mathrm{A}$, enolase 1 , and lactate dehydrogenase A gene promoters contain essential binding sites for hypoxia-inducible factor 1.J Biol Chem. 1996;271(51):32529-32537.

40. Firth JD, Ebert BL, Ratcliffe PJ. Hypoxic regulation of lactate dehydrogenase A. Interaction between hypoxia-inducible factor 1 and cAMP response elements. J Biol Chem. 1995;270(36):21021-21027.

41. Luo F, et al. Enhanced glycolysis, regulated by HIF-1 $\alpha$ via MCT-4, promotes inflammation in arsenite-induced carcinogenesis. Carcinogenesis. 2017;38(6):615-626.

42. Ullah MS, Davies AJ, Halestrap AP. The plasma membrane lactate transporter MCT4, but not MCT1, is up-regulated by hypoxia through a HIF-1alpha-dependent mechanism. J Biol Chem. 2006;281(14):9030-9037.

43. Ke X, et al. Hypoxia upregulates CD147 through a combined effect of HIF- $1 \alpha$ and Sp1 to promote glycolysis and tumor progression in epithelial solid tumors. Carcinogenesis. 2012;33(8):1598-1607.

44. Corcoran SE, O'Neill LA. HIF1 $\alpha$ and metabolic reprogramming in inflammation. JClin Invest. 2016;126(10):3699-3707.

45. Johnson TW, Wu Y, Nathoo N, Rogers JA, Wee Yong V, Dunn JF. Gray Matter Hypoxia in the Brain of the Experimental Autoimmune Encephalomyelitis Model of Multiple Sclerosis. PLoS ONE. 2016;11(12):e0167196.

46. Merezhinskaya N, Ogunwuyi SA, Mullick FG, Fishbein WN. Presence and localization of three lactic acid transporters (MCT1, -2, and -4) in separated human granulocytes, lymphocytes, and monocytes. J Histochem Cytochem. 2004;52(11):1483-1493.

47. Ke X, Chen Y, Wang P, Xing J, Chen Z. Upregulation of CD147 protects hepatocellular carcinoma cell from apoptosis through glycolytic switch via HIF-1 and MCT-4 under hypoxia. Hepatol Int. 2014;8(3):405-414.

48. Guglielmetti C, Najac C, Didonna A, Van der Linden A, Ronen SM, Chaumeil MM. Hyperpolarized 13C MR metabolic imaging can detect neuroinflammation in vivo in a multiple sclerosis murine model. Proc Natl Acad Sci U S A. 2017;114(33):E6982-E6991.

49. Tang W, Chang SB, HemLer ME. Links between CD147 function, glycosylation, and caveolin-1. Mol Biol Cell. 2004;15(9):4043-4050.

50. Bai Y, Huang W, Ma LT, Jiang JL, Chen ZN. Importance of N-glycosylation on CD147 for its biological functions. Int J Mol Sci. 2014;15(4):6356-6377.

51. Igakura T, et al. A null mutation in basigin, an immunoglobulin superfamily member, indicates its important roles in peri-implantation development and spermatogenesis. Dev Biol. 1998;194(2):152-165.

52. Naruhashi K, et al. Abnormalities of sensory and memory functions in mice lacking Bsg gene. Biochem Biophys Res Commun. 1997;236(3):733-737.

53. Igakura $\mathrm{T}$, et al. Roles of basigin, a member of the immunoglobulin superfamily, in behavior as to an irritating odor, lymphocyte response, and blood-brain barrier. Biochem Biophys Res Commun. 1996;224(1):33-36.

54. Choi IY, et al. A diet mimicking fasting pro- motes regeneration and reduces autoimmunity and multiple sclerosis symptoms. Cell Rep. 2016;15(10):2136-2146.

55. Mondal S, Pahan K. Cinnamon ameliorates experimental allergic encephalomyelitis in mice via regulatory T cells: implications for multiple sclerosis therapy. PLoS ONE. 2015;10(1):e0116566.

56. Balasubramanian S, Roselin P, Singh KK, Zachariah J, Saxena SN. Postharvest processing and benefits of black pepper, coriander, cinnamon, fenugreek, and turmeric spices. Crit Rev Food Sc Nutr. 2016;56(10):1585-1607.

57. Kannappan R, Gupta SC, Kim JH, Reuter S, Aggarwal BB. Neuroprotection by spice-derived nutraceuticals: you are what you eat! Mol Neurobiol. 2011;44(2):142-159.

58. Seki SM, et al. Lineage-specific metabolic properties and vulnerabilities of T cells in the demyelinating central nervous system. J Immunol. 2017;198(12):4607-4617.

59. Dang EV, et al. Control of T(H)17/T(reg) balance by hypoxia-inducible factor 1. Cell. 2011;146(5):772-784.

60. Haas R, et al. Lactate regulates metabolic and pro-inflammatory circuits in control of $\mathrm{T}$ cell migration and effector functions. PLoS Biol. 2015;13(7):e1002202.

61. Ranganathan P, et al. GPR81, a cell-surface receptor for lactate, regulates Intestinal homeostasis and protects mice from experimental colitis. Jimmunol. 2018;200(5):1781-1789.

62. Colegio OR, et al. Functional polarization of tumour-associated macrophages by tumour-derived lactic acid. Nature. 2014;513(7519):559-563.

63. Stone SC, et al. Lactate secreted by cervical cancer cells modulates macrophage phenotype. JLeukoc Biol. 2019;105(5):1041-1054.

64. Kornberg MD, et al. Dimethyl fumarate targets GAPDH and aerobic glycolysis to modulate immunity. Science. 2018;360(6387):449-453.

65. De Riccardis L, et al. Metabolic response to glatiramer acetate therapy in multiple sclerosis patients. BBA Clin. 2016;6:131-137.

66. Döring A, et al. Stimulation of monocytes, macrophages, and microglia by amphotericin B and macrophage colony-stimulating factor promotes remyelination. J Neurosci. 2015;35(3):1136-1148. 